\title{
Primary Mitochondrial Disorders of the Pediatric Central Nervous System: Neuroimaging Findings
}

Fabricio Guimarães Gonçalves, $M D$

César Augusto Pinheiro Ferreira Alves, $M D$

Beth Heuer, DNP, CPNP-PC

fames Peterson, MS, LCGC

Angela N.Viaene, $M D, P h D$

Sara Reis Teixeira, $M D, P h D$

Fuan Sebastián Martín-Saavedra, MD

Savvas Andronikou, MD, PhD

Amy Goldstein, MD

Arastoo Vossough, MD, PhD

Abbreviations: ATP $=$ adenosine triphosphate CNS $=$ central nervous system, $\mathrm{CoQ} 10=$ coen zyme Q10, FLAIR = fluid-attenuated inversion recovery, $\mathrm{LBSL}=$ leukoencephalopathy with brainstem and spinal cord involvement and lactate elevation, LHON = Leber hereditary optic neuropathy, MELAS = mitochondrial encephalopathy, lactic acidosis, and strokelike episodes, MERRF = myoclonic epilepsy with ragged-red fibers, $\mathrm{mtDNA}=$ mitochondrial DNA, $\mathrm{nDNA}=$ nuclear DNA, OXPHOS = oxidative phosphorylation, $\mathrm{PDH}=$ pyruvate dehydrogenase, $\mathrm{PMD}=$ primary mitochondrial disorder, $P O L G-\mathrm{RD}=$ polymerase $\gamma$-related disorder, tRNA $=$ transfer RNA

RadioGraphics 2020; 40:2042-2067

https://doi.org/10.1148/rg.2020200052

Content Codes: MR NR PD

From the Department of Radiology, Division of Neuroradiology (F.G.G., C.A.P.F.A., S.R.T., J.S.M.S., S.A., A.V.), Department of Pathology (A.N.V.), and Mitochondrial Medicine Frontier Program, Division of Human Genetics, Department of Pediatrics (B.H., J.P., A.G.), Children's Hospital of Philadelphia, 3401 Civic Center Blvd, Philadelphia, PA 19104-4399; and Department of Pediatrics (A.G.) and Radiology (S.A., A.V.), University of Pennsylvania Perelman School of Medicine (A.N.V.), Philadelphia, Pa. Presented as an education exhibit at the 2019 RSNA Annual Meeting. Received April 2, 2020; revision requested May 13 and received June 17; accepted June 26. For this journal-based SA-CME activity, the authors J.S.M.S. and A.G. have provided disclosures (see end of article). The other authors, the editor, and the reviewers have disclosed no relevant relationships. Address correspondence to F.G.G. (e-mail: goncalves.neuroradio@ gmail.com)

${ }^{\mathrm{C}}$ RSNA, 2020
Primary mitochondrial disorders (PMDs) constitute the most common cause of inborn errors of metabolism in children, and they frequently affect the central nervous system. Neuroimaging findings of PMDs are variable, ranging from unremarkable and nonspecific to florid and highly suggestive. An overview of PMDs, including a synopsis of the basic genetic concepts, main clinical symptoms, and neuropathologic features, is presented. In addition, eight of the most common PMDs that have a characteristic imaging phenotype in children are reviewed in detail.

Online supplemental material is available for this article.

${ }^{\circ}$ RSNA, $2020 \cdot$ radiographics.rsna.org

\section{SA-CME LEARNING OBJECTIVES}

After completing this journal-based SA-CME activity, participants will be able to:

- Describe the most typical imaging findings and clinical manifestations of PMDs that have a characteristic imaging phenotype in children.

- Discuss the gamut of imaging findings that may occur in PMDs.

- Discuss conditions that mimic PMDs.

See rsna.org/learning-center-rg.

\section{Introduction}

Mitochondria are complex intracellular double-membrane organelles that are present in all nucleated cells (Fig 1). In humans, mitochondria are involved in several vital processes, including generation of adenosine triphosphate (ATP) by means of oxidative phosphorylation (OXPHOS), redox homeostasis, regulation of calcium homeostasis, fatty acid oxidation, biogenesis of heme and iron-sulfur proteins, and programmed cell death (ie, apoptosis) (1). The OXPHOS complex is located on the inner mitochondrial membrane and consists of five main enzymatic complexes. These five complexes make up the mitochondrial respiratory chain (complexes I-IV) and ATP synthase pump (complexV) (2) (Fig 1).

Primary mitochondrial disorder (PMD) is caused by pathogenic variants in mitochondrial DNA (mtDNA) or nuclear DNA (nDNA) that commonly affect the pediatric central nervous system (CNS), lacking pathognomonic imaging findings. At neuroimaging, PMD findings vary widely. Certain PMDs have typical neuroimaging features (typical spatial distribution) and may evolve during the course of the disease (temporal distribution).

In the appropriate clinical setting, the following imaging features may indicate the possibility of a PMD: neuroimaging combinations of signal intensity changes in the basal ganglia, thalamus, brainstem, cortex, white matter (leukodystrophy), cerebellum, or spinal cord; calcification (especially in the basal ganglia); white matter cavitation; cerebral and cerebellar atrophy; myelination delay or 


\section{TEACHING POINTS}

- Primary mitochondrial disorder (PMD) is caused by pathogenic variants in mitochondrial DNA (mtDNA) or nuclear DNA (nDNA) that commonly affect the pediatric central nervous system (CNS), lacking pathognomonic imaging findings.

- PMDs are clinically heterogeneous, may occur at any age, and manifest with a broad range of multiple symptoms. They can affect any organ or tissue but usually affect those organs or tissues that are highly dependent on aerobic metabolism and that have high energy requirements, such as the CNS, heart tissue, and skeletal muscle.

- Impaired ATP production is the common outcome of all PMDs. It is well known that certain neuronal populations, including large and long projection neurons (particularly those with sparsely myelinated axons) and fast-spiking interneurons, are especially dependent on oxidative metabolism and extremely susceptible to respiratory chain impairment.

- Common CNS PMDs with characteristic imaging phenotypes include Leigh syndrome, POLG-RDs, MELAS, Kearns-Sayre syndrome, Leber hereditary optic neuropathy (LHON), pyruvate dehydrogenase (PDH) complex deficiency, coQ10 deficiency, and leukoencephalopathy with brainstem and spinal cord involvement and lactate elevation (LBSL).

- The spectrum of MR neuroimaging findings of PMDs includes basal ganglia signal intensity changes, basal ganglia calcification, cortical signal intensity changes and malformations, subependymal cysts, and white matter changes (leukoencephalopathy, white matter cavitation, callosal agenesis or dysgenesis, and delayed myelination or hypomyelination).

hypomyelination; callosal agenesis or dysgenesis; subependymal cysts; optic nerve atrophy; cerebral edema (cytotoxic and vasogenic); and certain abnormal MR spectroscopy findings (lactate and succinate peaks).

\section{Genetics of Mitochondrial Disease}

The human mitochondrial genome is a genetically compact, circular double-stranded DNA molecule that is 16569 base-pairs in length, and there are typically between 100 and 10000 copies per cell (3). According to the updated human MitoCarta (MitoCarta2.0) (4), the most comprehensive inventory of human genes that encode mitochondrial proteins, there are 1158 gene-encoding proteins involved in mitochondrial metabolism. The human mitochondrial proteome comprises approximately 1500 distinct proteins within the mitochondria, only 37 of which are encoded by mtDNA (5). In addition to the 13 mtDNA-encoded proteins that make up key subunits in the respiratory chain, mtDNA also encodes machinery for mtDNA transcription and translation, including 22 transfer RNAs (tRNAs) and two ribosomal RNAs (rRNAs).

In total, 13 polypeptides are inserted into the inner mitochondrial membrane, where they form the core; membrane-bound subunits of respiratory chain complexes I, III, and IV; and ATP synthase (3). As an exception, membrane-bound subunits of respiratory chain complex II, which is entirely encoded by nDNA, are not formed by these polypeptides.

Variations in mtDNA or nDNA can cause PMDs, which are considered the most common causes of inborn errors of metabolism. In 1988, two pioneer studies published by Holt et al (6) and Wallace et al (7) demonstrated that variants in mtDNA are associated with human diseases. Since the publication of these seminal studies, more than 350 individual genetic diseases have been linked to the umbrella term primary mitochondrial disorder, which typically affects multiple organs and systems, causing an average of 16 symptoms in each individual (8). The most common CNS symptoms include vision loss, ophthalmoplegia, ptosis, sensorineural hearing loss, hypotonia, epilepsy, headache, stroke, peripheral neuropathy, global developmental delay, and intellectual disability.

PMDs represent an extensive and highly diverse group of genetic conditions that often have impaired energy production in common. PMDs can be classified on the basis of disrupted pathways, including the following anomalies: (a) OXPHOS dysfunction (affecting components of the respiratory chain subunits and assembly factors), (b) mtDNA maintenance disorders (affecting mtDNA nucleotide pool, replication, maintenance, and transcription), (c) mitochondrial translation defects (affecting mitochondrial tRNA, aminoacyl-tRNA synthetases, tRNA modification, mitochondrial rRNA, RNA processing, mitoribosome subunits and assembly, and protein synthesis), (d) mitochondrial quality control defects (affecting the phospholipid membrane, import machinery, and mitochondrial dynamics), (e) metabolic defects (affecting the tricarboxylic acid cycle, pyruvate metabolism, fatty acid metabolism, and coenzyme A metabolism), $(f)$ vitamin and cofactor metabolism defects (affecting coenzyme Q10 [coQ10] biosynthesis; iron-sulfur cluster protein biosynthesis; lipoic acid biosynthesis; and metabolism of biotin, thiamine, heavy metal, and riboflavin), and (g) other cellular defects associated with mitochondrial dysfunction (affecting calcium homeostasis, heme biosynthesis, DNA repair, apoptosis, and miscellaneous functions) (9). A comprehensive list of genes that are organized according to their disrupted pathways is published in the literature (9).

PMDs can manifest with any inheritance pattern, including autosomal dominant, autosomal recessive, and X-linked inheritance for nDNA variants and maternal inheritance for mtDNA variants (8). They may also occur as sporadic (or de novo) diseases. There are many 
Figure 1. Mitochondria are cytoplasmic organelles composed of an inner and outer membrane, with an intervening intermembrane space. The mitochondrial matrix lies within the inner mitochondrial membrane, which is highly infolded (to increase the amount of surface area) to form the crista on which OXPHOS occurs. During OXPHOS, energy is transferred as electrons in the presence of oxygen to generate chemical energy in the form of adenosine triphosphate (ATP). This aerobic respiration process occurs within the five complexes of the respiratory chain, each of which has multiple protein subunits that must be properly assembled. Thirteen of these subunits are encoded by the mtDNA, which constitutes the core structure of respiratory chain complexes I, III, IV, and V. The remaining respiratory chain subunits in all five complexes are encoded by the nDNA genes and imported into the mitochondria. mtDNA is a small circular genome of 16569

base pairs with 37 genes. Thirteen of these 37 genes are protein-coding genes. The remaining 24 genes encode the RNA needed for synthesis of the protein-coding mtDNA genes, including two rRNAs and 22 tRNAs. The mtDNA is located within the mitochondria attached to the inner mitochondrial membrane within the mitochondrial matrix. $A D P=$ adenosine diphosphate, $F A D=$ flavin adenine dinucleotide, $F A D H=$ flavin adenine dinucleotide plus hydrogen, $N A D=$ nicotinamide adenine dinucleotide, $N A D H=$ nicotinamide adenine dinucleotide plus hydrogen, $P_{i}=$ inorganic phosphate. (Reprinted, with permission, from The Children's Hospital of Philadelphia, (02020, all rights reserved.)

copies of the mtDNA per mitochondrion and many mitochondria per cell. The exact numbers depend on the given organ's energy requirements and therefore differ among organs in the human body. Not all of the mtDNA copies in an individual may be abnormal; therefore, mtDNA variants exist in a state ranging from heteroplasmic to homoplasmic. Heteroplasmy, also referred to as the mutation load, describes the proportion of the mitochondrial genome that has a pathogenic variant compared to the reference allele at that locus. Homoplasmy indicates that a particular variant at a certain position is found in every copy of the mtDNA; thus, the mtDNA is uniform at that position for the variant. Heteroplasmy levels can vary greatly between family members and within a given individual's different tissues (8). The phenomenon of heteroplasmy may greatly influence the range of clinical manifestations in patients with PMDs (8). In general, the percentage of mtDNA that harbors a pathogenic variant (heteroplasmy) that is, a higher mutation load-correlates directly with disease severity (10).

\section{Clinical Symptoms}

PMDs are clinically heterogeneous, may occur at any age, and manifest with a broad range of multiple symptoms. They can affect any organ or tissue but usually affect those organs or tissues that are highly dependent on aerobic metabolism and that have high energy requirements, such as the CNS, heart tissue, and skeletal muscle. These disorders are often relentlessly progressive, with high morbidity and mortality. In a survey involving a cohort of children and adult patients (11), those with PMDs reported an average of 16 overall symptoms, highlighting the substantially varied burden of disease. In pediatric patients, the more common symptoms and findings include decreased vision, retinal abnormalities, optic neuropathy, eye muscle abnormalities (ophthalmoplegia), ptosis, sensorineural hearing loss, speech abnormalities, sleep apnea, hypotonia, epilepsy, headache, stroke, peripheral neuropathy, global developmental delay, intellectual disability, and learning disabilities. Other more common symptoms and findings include difficulty sleeping, behavior problems, autism spectrum disorder, mood disorders, balance problems, heart arrhythmias, cardiomyopathy, hypercholesterolemia, difficulty gaining weight, gastrointestinal abnormalities, liver disease, kidney disorders, muscle weakness, exercise intolerance, dehydration, dysautonomia, and chronic fatigue (11). The onset or relapse of a PMD may be triggered 


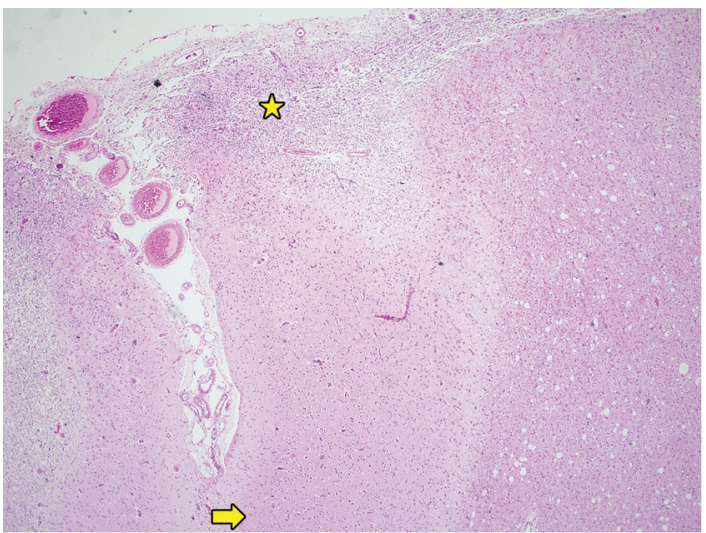

a.

Figure 2. (a) Low-magnification photomicrograph of a cortex tissue specimen obtained at brain autopsy in a 16-yearold boy with MELAS shows an infarct-like lesion (ఓ) involving the gyral crest. The depths of the sulci are generally spared (arrow). (Hematoxylin-eosin stain; original magnification, $\times 2$.) (b) Higher-magnification photomicrograph of an infarct lesion in the cortex of another patient who has MELAS shows tissue rarefaction and macrophage collections (right side) and surrounding reactive gliosis (left side). (Hematoxylin-eosin stain; original magnification, $\times 10$.)

by various stressors such as infection. Despite the extensive range of symptoms and disabilities, there is no U.S. Food and Drug Administration-approved treatment for PMDs. Rather, the treatment remains predominantly empirical and based on the symptoms.

\section{Neuropathologic Findings}

Similar to the heterogeneous imaging and clinical features, the neuropathologic findings of PMDs also vary across these disorders. Impaired ATP production is the common outcome of all PMDs. It is well known that certain neuronal populations, including large and long projection neurons (particularly those with sparsely myelinated axons) and fast-spiking interneurons, are especially dependent on oxidative metabolism and extremely susceptible to respiratory chain impairment. The main neuropathologic features of PMDs include neuronal damage, vasculonecrotic changes, and spongy degeneration. Gliosis and demyelination also are frequently reported (12). Neuronal changes may range from diffuse atrophy to neuronal loss. Vasculonecrotic lesions are mainly characterized by focal tissue rarefaction, gliosis, and/or cavitation (13). These changes are not specific to an individual PMD; however, they usually manifest with a distinctive distribution and different degrees of severity, which are often typical of a specific disorder (13).

Mitochondrial encephalopathies can affect both the gray and white matter. Gray matter lesions are often multifocal, mainly identified by vasculonecrotic lesions and neuronal loss, while white matter lesions are characterized by demyelination and spongy degeneration. Gliosis can be found in both gray and white matter, with various degrees of severity.
Diseases that predominantly affect the gray matter include mitochondrial encephalopathy, lactic acidosis, and strokelike episodes (MELAS); myoclonic epilepsy with ragged-red fibers (MERRF); polymerase $\gamma$-related disorders (POLG-RDs); and Leigh syndrome (13). Gray matter involvement in MELAS is typically multifocal, with infarct-like lesions in the occipital, parietal, and temporal cortices. These lesions are associated with neuronal loss and gliosis; gyral crests are typically more extensively affected than are sulci (Fig 2). Neuronal loss is also seen in the basal ganglia, thalamus, cerebellum, and brainstem. Mineral deposits are sometimes present in blood vessels of the basal ganglia, and capillary proliferation may be seen around infarct-like lesions (14). Although the vasculonecrotic lesions may resemble ischemic areas, they do not respect vascular territories. Therefore, vascular mitochondrial dysfunction and proliferation can be important in the pathogenesis of strokelike episodes (15). Neuronal loss of either subcortical gray matter structures or brainstem and spinal cord nuclei occurs. It is not yet known whether these changes are secondary to primary cortical events or this condition should be considered a multisystem degeneration (13).

Neuropathologic studies of MERRF (13) show multisystem degeneration with neuronal loss mainly in subcortical structures-that is, in the dentate nucleus and brainstem and especially the inferior olivary nuclei-and degeneration of the cerebellar cortex and sometimes in the Clarke nuclei and dorsal root ganglia (13).

In POLG-RDs, the hemispheric cortex (the occipital lobe-calcarine cortex in particular), the thalamus, and to a lesser extent the cerebellar cortex are the main sites of damage, which is characterized by necrotizing lesions (microcystic 


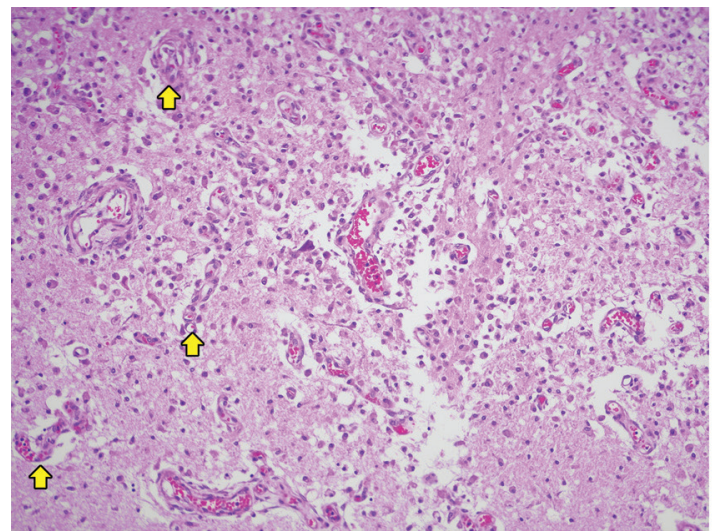

a.

Figure 3. Leigh syndrome lesion in a 14-month-old girl. (a) Photomicrographic section of the brainstem obtained at autopsy shows a vasculonecrotic lesion containing numerous macrophages, rarefied brain parenchyma, and reactive vessels (arrows). (Hematoxylin-eosin stain; original magnification, $\times 10$.) (b) Photomicrographic section of the brainstem from the same child shows an older Leigh syndrome lesion with cavitation and surrounding gliosis (right side). (Hematoxylin-eosin stain; original magnification, $\times 10$.)

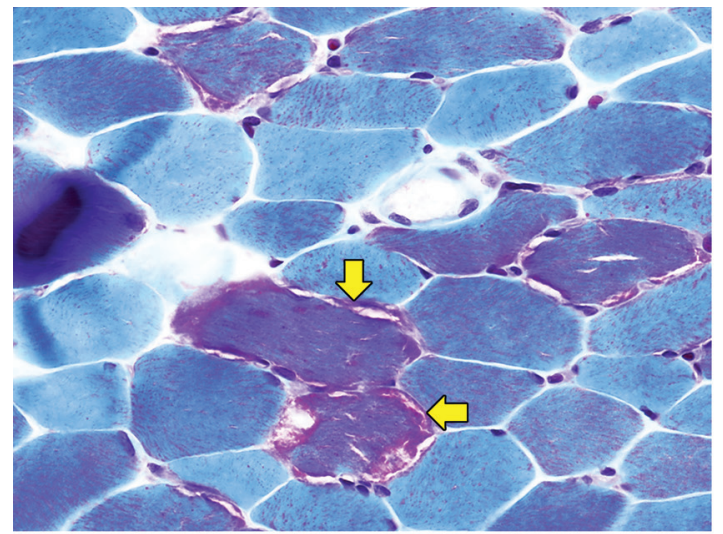

a.

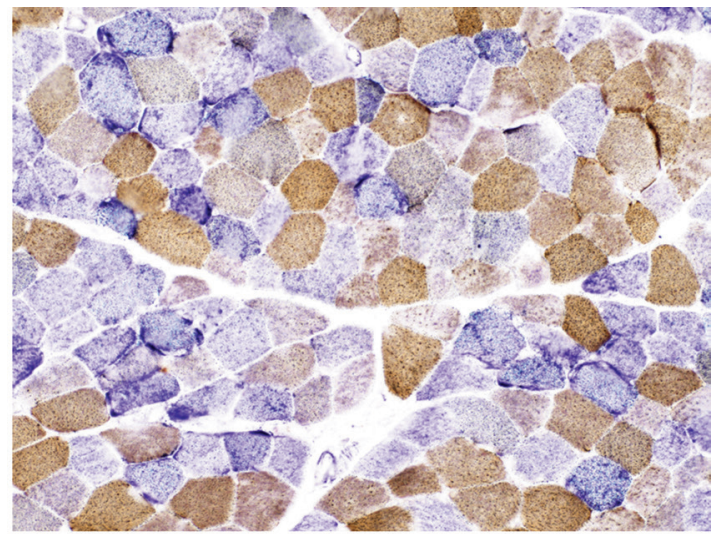

b.

Figure 4. Suspected Kearns-Sayre syndrome in a 17-year-old adolescent girl. (a) Photomicrograph of a Gomori trichrome-stained muscle biopsy specimen shows multiple ragged red fibers (arrows). (Original magnification, $\times 40$.) (b) Photomicrograph of a muscle biopsy specimen stained with combined cytochrome c oxidase-succinate dehydrogenase shows numerous cytochrome c oxidase-negative fibers (appearing bright blue) that were positive for succinate dehydrogenase. The fibers with retained cytochrome $c$ oxidase stain appear brownish gray. (Original magnification, $\times 20$.)

degeneration, capillary proliferation, different cell death patterns, and marked gliosis) and severe neuronal loss. Gliosis of the hemispheric white matter also may be present (16).

Leigh syndrome-related neuropathologic changes are characterized by bilateral and symmetric vasculonecrotic lesions, which are preferentially localized in the basal ganglia, thalamus, and brainstem (12). Lesions are often of varying ages, as reflected in their histopathologic appearances. Recently manifesting lesions may contain edema, eosinophilic neurons, and a few macrophages, whereas somewhat older lesions are characterized by numerous macrophages, tissue rarefaction, gliosis, and reactive vessels (Fig 3a). The oldest lesions resemble chronic infarcts, with cavitation and surrounding gliosis (Fig $3 b$ ). Spongy degeneration and capillary proliferation in the cerebral cortex and Purkinje cell loss also have been described (12).

The neuropathologic hallmark finding of Kearns-Sayre syndrome is spongy degeneration of the hemispheric white matter and brainstem fiber tracts. Neuronal loss of the gray nuclei of the brainstem and Purkinje cell layer also has been described (12). Mineralization of the blood vessel walls in the deep gray nuclei may be present. Severe white matter involvement featuring a leukodystrophy-like pattern is observed in the rare early-onset conditions associated with complex I and complex II deficiencies (17).

Classic muscle biopsy findings of mitochondrial disease include ragged red fibers seen on Gomori trichrome-stained specimens owing to subsarcolemmal collections of mitochondria (Fig $4 a)$. Likewise, ragged blue fibers may be seen on 


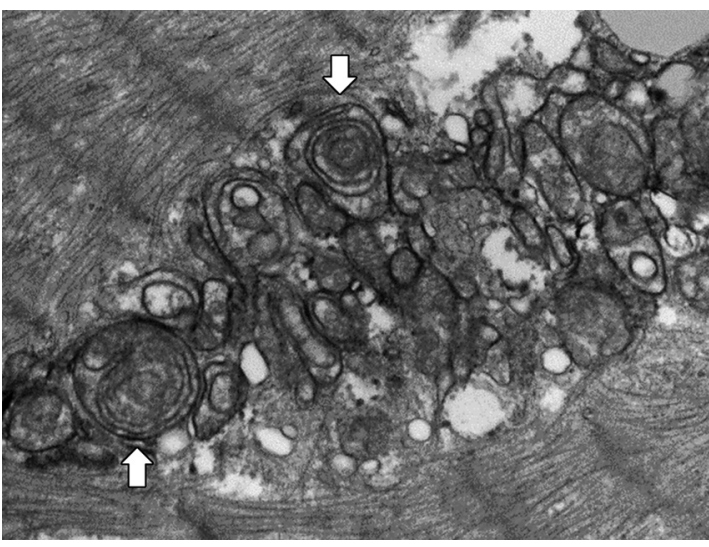

Figure 5. Electron micrograph of a muscle biopsy specimen from a 3-year-old boy with a variant in POLG and multiple mtDNA deletions. Mitochondrial cytopathy, including abnormal accumulations of mitochondria and mitochondria with concentric cristae (arrows), are seen.

succinate dehydrogenase- and nicotinamide adenine dinucleotide-stained specimens. Additional findings include cytochrome c oxidase-negative fibers, which stain positively for succinate dehydrogenase (Fig 4b). At electron microscopic examination, mitochondria may be enlarged and/ or show abnormal accumulations. Abnormal cristae (eg, concentric cristae) and paracrystalline inclusions are additional findings that may be present (Fig 5).

\section{Common CNS PMD Phenotypes}

Common CNS PMDs with characteristic imaging phenotypes include Leigh syndrome, POLGRDs, MELAS, Kearns-Sayre syndrome, Leber hereditary optic neuropathy (LHON), pyruvate dehydrogenase (PDH) complex deficiency, coQ10 deficiency, and leukoencephalopathy with brainstem and spinal cord involvement and lactate elevation (LBSL). The genetic features, patient age at PMD onset, and typical clinical manifestions and imaging findings for each of the listed PMDs are summarized in Table E1. Several other less common PMDs are briefly mentioned later, in the differential diagnosis sections, but they are not described in depth owing to space constraints.

\section{Leigh Syndrome}

Definition.-Leigh syndrome, also known as subacute necrotizing encephalopathy, represents the most common phenotype of PMDs, especially in the pediatric population. Leigh syndrome has an estimated incidence of one case per 40000 live births, with higher incidences in specific populations owing to founder mutations (18). The diagnostic criteria for Leigh syndrome include neuroimaging evidence of bilateral basal ganglia and/or brainstem lesions in association with developmental delay and/or regression in early childhood, elevated lactate levels in blood or CSF, and/or a confirmed molecular cause (19).

Genetic Features.-Leigh syndrome has a variable mode of inheritance. Inheritance may occur in an autosomal recessive pattern (most common), mitochondrial DNA inheritance pattern (maternal), or X-linked recessive pattern (less common), or even sporadically (20). Pathogenic variants causing Leigh syndrome have been identified in more than 95 genes across both nDNA and mtDNA genomes, with regular ongoing identification of novel genetic disorders owing to advances in next-generation sequencing (9).

Leigh syndrome can be divided into two categories: $\mathrm{nDNA}$ associated and mtDNA associated. In the nDNA category, the most common biochemical defect is complex I deficiency; variants affecting the NDUFS4 (nicotinamide adenine dinucleotide plus hydrogen-dehydrogenase) gene subunit are the most frequent cause of this deficiency (21). In patients with Leigh syndrome who have NDUFS4 variants, the initial manifestation of Leigh syndrome occurs at around age 8 months, with a rapidly progressive course. Death occurs by age 30 months (21). Nuclear gene mutations affecting complex II and complex III, and ubiquinone deficiencies are rare causes of Leigh syndrome and include mutations in $S D H A, S D H B$, and $S D H A F 1$ (complex II); UQCRQ, BCS1L, and TTC19 (complex III); and COQ9 and PDSS2 (ubiquinone) (20).

Disruptions of complex IV constitute approximately $15 \%$ of Leigh syndrome cases (22). The most common cause of Leigh syndrome due to complex IV deficiency is $S U R F 1$ gene variants. Patients with $S U R F 1$ variants tend to survive longer (median age at death, 5.4 years) than those with Leigh syndrome due to other genetic causes (23). Other causes of nuclear Leigh syndrome (such as PDH complex deficiency) are discussed later in this article.

Several mtDNA genes have been associated with Leigh syndrome, including six genes that encode for complex I subunits (ND1, ND2, ND3, ND4, ND5, and ND6), one that encodes for complex IV subunits (CO3), and two that encode for complex $\mathrm{V}$ subunits (ATP and ATP8). Of these genes, ND3, ND5, and ATP6 are the leading causes of mtDNA-associated Leigh syndrome. Several mt-tRNAs, such as the MT-TK gene, have also been associated with Leigh syndrome. ATP6 ( $\mathrm{m} .8993 \mathrm{~T}>\mathrm{G}$ or $\mathrm{m} .8993 \mathrm{~T}>\mathrm{C}$ ) is believed to be the culprit in approximately one in 10 cases in this Leigh syndrome category (22). 
Clinical Features.-The clinical symptoms of individuals with Leigh syndrome are variable but commonly manifest by age 2 years (19). Acute exacerbations or relapses are frequently observed in the first year of the disease and are triggered by stressors such as infection, fever, anesthesia or surgery, or dehydration. The most common symptoms include hypotonia, regression of milestones, and/or developmental delay. Other frequently observed neurologic signs are caused by brainstem and basal ganglia lesions and include strabismus, nystagmus, swallowing difficulties, chorea, dystonia, ataxia, pyramidal signs, and respiratory insufficiency. The overall survival rate for individuals with Leigh syndrome is typically poor, with death often occurring by age 6 years; however, some affected persons may live much longer (22). The most important outcome predictors are age at onset younger than 6 months, brainstem involvement, failure to thrive, and necessity of intensive care.

Imaging Findings. - The most common imaging findings observed in individuals with Leigh syndrome are bilateral lesions in the basal ganglia, especially in the striatum (caudate nucleus and putamen), diencephalon (mainly the medial aspect of the thalamus), and brainstem (substantia nigra, oculomotor nuclei, periaqueductal gray matter, and inferior olivary nuclei) (Fig 6). Less common locations include those in the white matter, cortex, cerebellum, and spinal cord (24). The lesions are usually hyperintense on T2-weighted and FLAIR MR images and hypointense on T1-weighted MR images, with or without restricted diffusion. Areas of enhancement, foci of calcification, and necrosis also can be variably observed. An abnormally elevated lactate peak also is commonly seen in these individuals at $\mathrm{MR}$ spectroscopy, especially when the regions of interest are located in the involved areas and during exacerbation of the disease. The typical temporal evolution at imaging in a patient with Leigh syndrome is shown in Figure 7.

Although the literature contains several imaging descriptions of Leigh syndrome, it contains no description of a standardized assessment of neuroimaging features in association with particular genes and metabolic pathways. At neuroimaging, complex I deficiency may involve bilateral symmetric basal ganglia lesions, and approximately one-third of cases involve hypertrophic cardiomyopathy (25). A few descriptions highlighting the pathogenic variants of the SURF1 gene are available. Subjects with a particular pattern of lesion distribution affecting the brainstem, including the inferior olivary nuclei, restiform bodies, and dentate nuclei sparing of the basal ganglia, have been classically described as having this gene related to Leigh syndrome (26).

Although there may be some heterogeneity in the imaging appearance of $S U R F 1$-associated Leigh syndrome, these variants often spare the putamina and involve the brainstem to a greater extent. At neuroimaging, patients with SURF1associated Leigh syndrome may have symmetric T2-hyperintense lesions in the brainstem and/ or basal ganglia, leukoencephalopathy (lesions predominantly affecting the cerebral white matter), cerebellar atrophy, and/or involvement of the dentate nucleus (23).

Differential Diagnosis.—The differential diagnosis of Leigh syndrome is extensive. The main disorders associated with signal intensity changes in the basal ganglia, thalamus, and/or brainstem are summarized in Table 1.

\section{POLG-RDs}

Definition.-POLG is a nuclear gene that encodes DNA polymerase $\gamma$, which is responsible for replication of the mitochondrial genome and considered the only human DNA polymerase that is active during mtDNA replication (27). $P O L G$ pathogenic variants represent the most prevalent single-gene cause of PMD. The classically reported prototypical clinical phenotypes associated with $P O L G$ pathogenic variants are Alpers-Huttenlocher syndrome; myocerebrohepatopathy spectrum; myoclonic epilepsy myopathy sensory ataxia; ataxia neuropathy spectrum, which previously was also called mitochondrial recessive ataxia syndrome, spinocerebellar ataxia with epilepsy, or sensory ataxia neuropathy dysarthria and ophthalmoplegia; autosomal recessive progressive external ophthalmoplegia; and autosomal dominant progressive external ophthalmoplegia. However, individuals with $P O L G$-RDs may not fit the profile for a specific entity, as there is a broad and sometimes overlapping clinical spectrum (28). Alpers-Huttenlocher syndrome is reported to affect approximately one in 51000 people (29).

Genetics.-POLG-RDs are typically inherited in an autosomal recessive manner. However, autosomal dominant inheritance also occurs, usually owing to a mutation in the polymerase domain of POLG. POLG pathogenic variants lead to errors in mtDNA replication, resulting in multiple small mtDNA deletions, an overall reduction in the number of mtDNA genome copies ( $\mathrm{mtDNA}$ depletion), and decreased ATP production, and thus leading to chronic loss of cellular energy (30). 


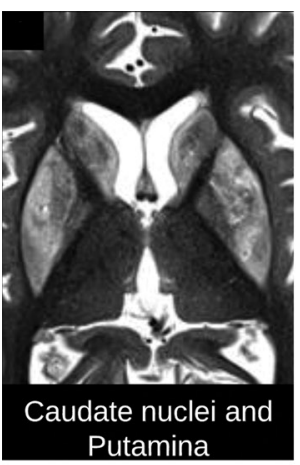

a.

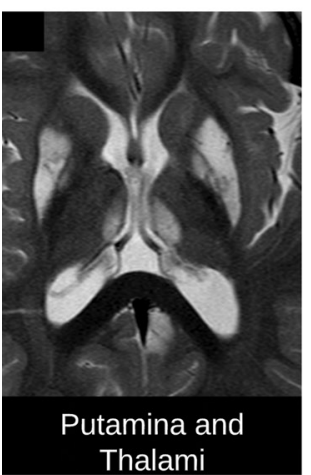

b.

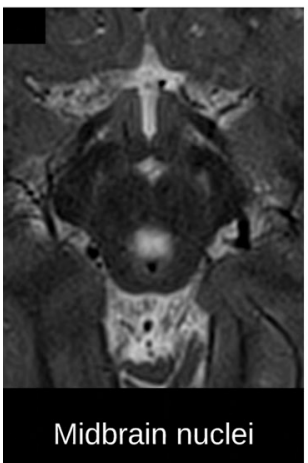

g.

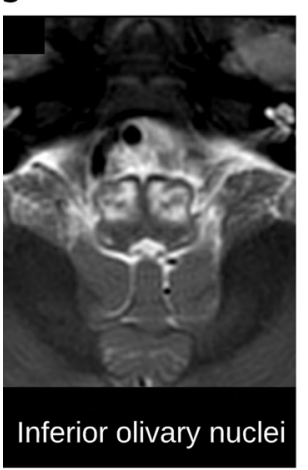

I.

Figure 6. Spectrum of brain abnormalities in patients with Leigh syndrome. (a-d) Axial (a-c) and coronal (d) MR images show typical Leigh syndrome lesions in the basal ganglia and thalami, including bilateral and symmetric hyperintense lesions in the caudate nuclei and putamina (a), bilateral putamina and thalami (b), and thalami (d) on T2-weighted images, and in the bilateral globi pallidi on the fluidattenuated inversion-recovery (FLAIR) image (c). (e-h) Axial T2-weighted MR images show typical Leigh syndrome lesions in the midbrain, including hyperintense lesions surrounding the periaqueductal gray matter (e), and in the midbrain tegmentum (f), bilateral oculomotor nuclei (g), and bilateral substantia nigra (h). (i-m) Axial (i, k, l, m [bottom]) and coronal (j, $\mathbf{m}$ [top]) MR images show Leigh syndrome lesions in the posterior fossa, including hyperintense lesions in the pontine tegmentum (i), cerebellar cortex (with atrophy) (j), bilateral inferior olivary nuclei $(I)$, and bilateral dentate nuclei $(m)$ on T2-weighted images, and in the medulla on the FLAIR image (k). (n-p) Axial $(n, 0)$ and sagittal (p) MR images show atypical hyperintense Leigh syndrome lesions in the cortex on the FLAIR image (n), and in the bilateral white matter (with cavitation) (o) and spinal cord (extensively) (p) on T2-weighted images.

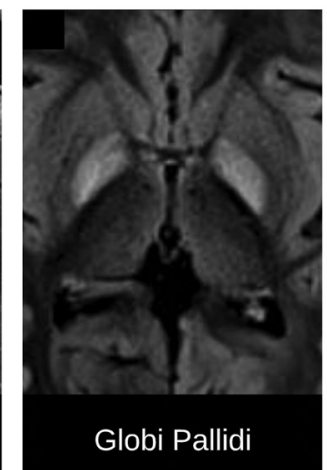

c.

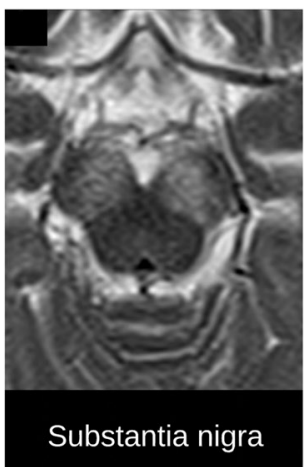

h.

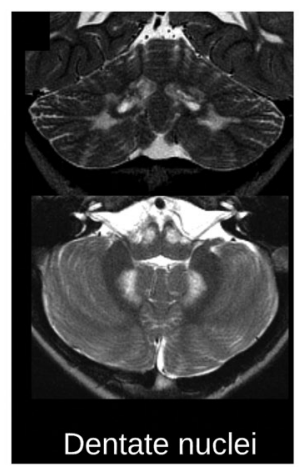

$\mathrm{m}$.

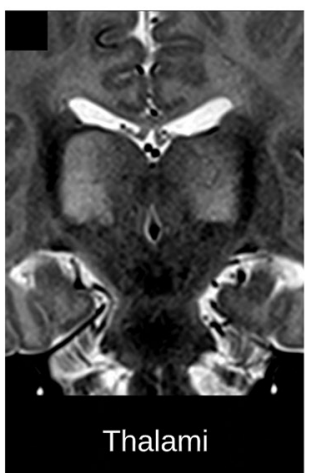

d.

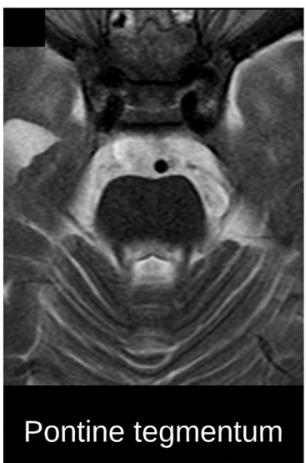

i.

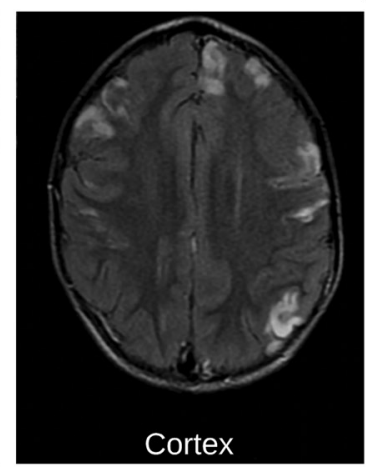

n.

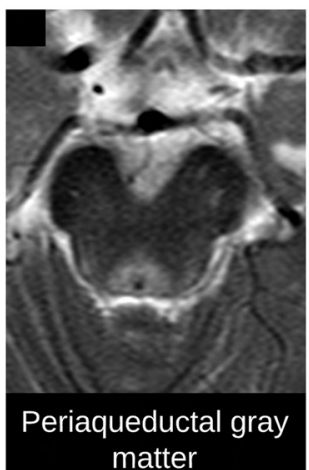

e.

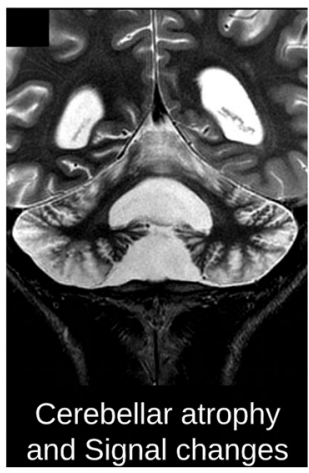

j.

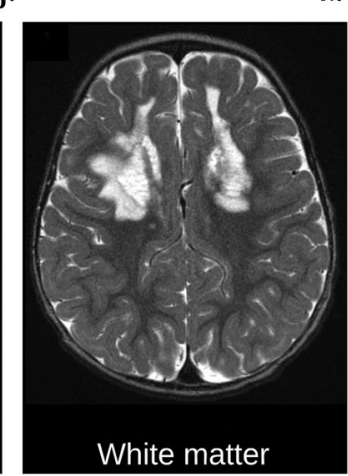

o.

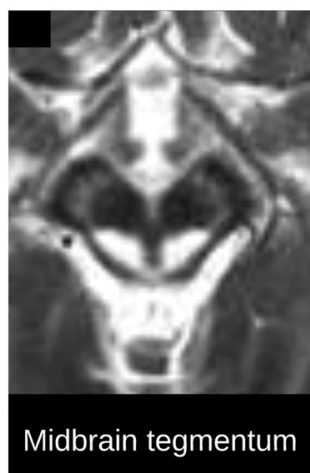

f.

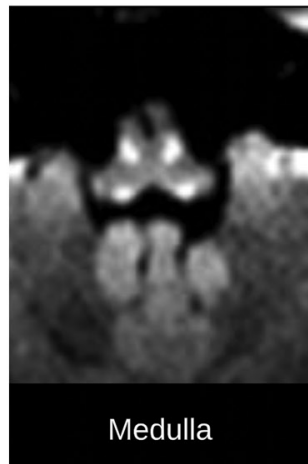

k.

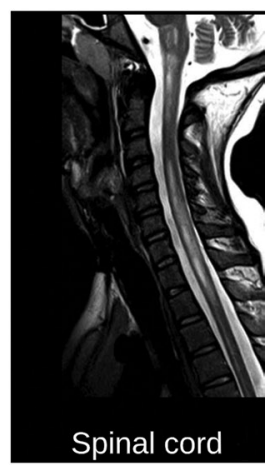

p.
Clinical Features.-POLG-RDs are considered the most common cause of mitochondrial epilepsy (31). Patient ages at onset of $P O L G$-RDs range from infancy to late adulthood and have a major role in the type of clinical feature and outcome. Children with POLG-RDs typically have seizures, which may be the first clinical manifestation in $50 \%$ of affected patients (32). The critical factor that triggers epileptic activity is mtDNA depletion. mtDNA depletion causes loss of respiratory chain components, which restricts normal energy metabolism, causing a 


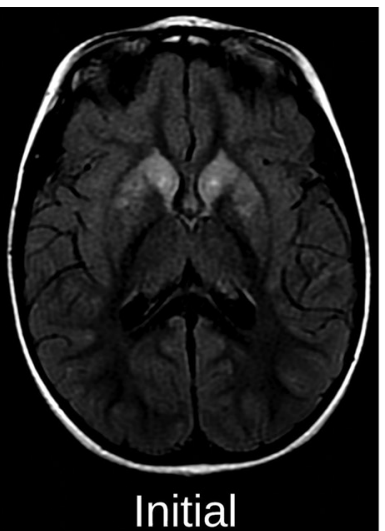

a.

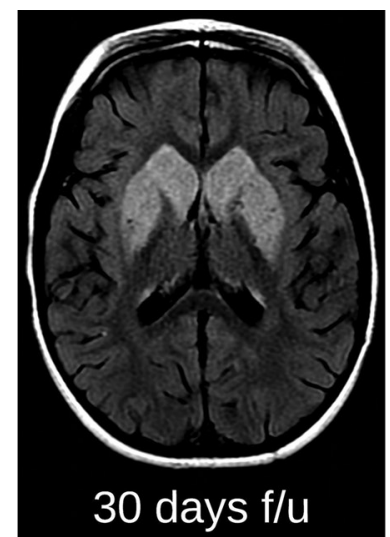

b.

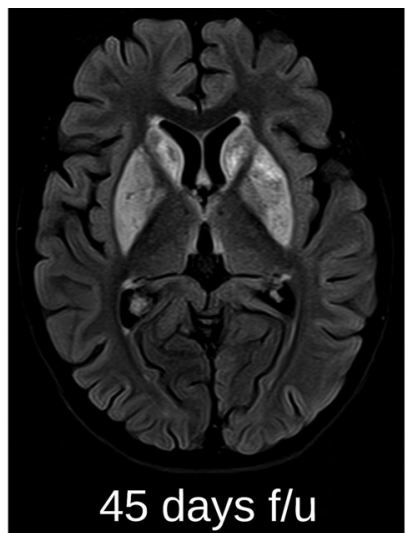

c.

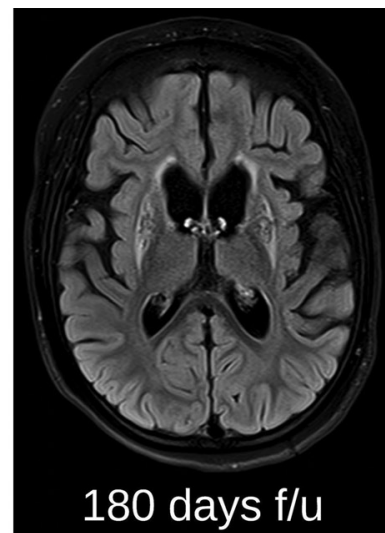

d.

Figure 7. Axial FLAIR MR images demonstrate temporal evolution at four time points in a 3-year-old girl with Leigh syndrome. (a) Initial image shows signal intensity changes involving mainly the caudate nuclei heads. (b) Follow-up (f/u) image shows marked edema involving the caudate nuclei heads and putamina. (c) Subsequent follow-up image shows resolution of the mass effect and edema, with maintenance of the striatal signal intensity changes. (d) Late follow-up image shows that burnout of the bilateral putamen and caudate is evident, with volume loss and ill-defined signal intensity changes associated with moderate ventriculomegaly and diffuse mild sulcal effacement.

continued neurodegenerative process that interferes with neuronal function. This cycle ultimately leads to neuronal death and parenchymal necrosis (31). In addition to seizures, patients may have ophthalmoplegia, ataxia, regression of neurologic symptoms, and liver disease. Valproic acid, an effective anticonvulsant for treatment of epilepsy, is contraindicated in cases of suspected and confirmed POLG-RD owing to the increased risk of precipitating and/or accelerating liver failure. Hence, sequencing and deletion or duplication analysis of $P O L G$ gene activity is currently the best diagnostic approach to preventing valproic acid-induced liver failure and patient death (33).

Imaging Findings.-Strokelike changes, with increased signal intensity at T2-weighted and FLAIR MR imaging, were the most common imaging findings described in a comprehensive literature review of data on 136 patients who had epilepsy with $P O L G$-RDs, which were more common in the occipital lobes (32). These lesions may show cytotoxic, vasogenic, or mixed changes (depending on the timing of the imaging in relation to onset of symptoms) accompanied by hemorrhage. Other involved structures (parietal, temporal, and frontal lobes; thalami [often bilateral]; basal ganglia; and cerebellum) showed increased T2 signal intensity. Unusual imaging appearances such as enhancement of bilateral cranial nerves (III, $\mathrm{V}-\mathrm{X}$ ) and cervical nerve roots, and $\mathrm{T} 2$ hyperintensity of the inferior colliculus have been reported. Overall, signal intensity abnormality of the brainstem is extremely uncommon (32). Early imaging findings of $P O L G$-RDs at T2-weighted, FLAIR, and diffusion-weighted MR imaging may include unilaterally or bilaterally increased signal intensity changes in the perirolandic cortex (perirolandic sign), unilateral or bilateral thalamic signal intensity changes, and/or signal intensity changes in the bilateral white matter (leukoencephalopathy), which may show restricted diffusion. At followup MR imaging, a variable degree of volume loss is typical and may be accompanied by thalamic signal intensity changes and encephalomalacia of the occipital lobes (Fig 8) (34).

\section{Mitochondrial Encephalomyopathy, Lactic Acidosis, and Strokelike Episodes}

Definition.-According to the most recent clinical criteria published by the MELAS Study Group Committee in Japan (35), MELAS manifestations may be assigned to category A or category B (Table 2). For a definitive diagnosis of MELAS, two features each from category A and category B are required (four features or more). For a diagnosis of suspected MELAS, one feature from category A and two features from category $\mathrm{B}$ are necessary (at least three features). MELAS has an estimated prevalence of 10-15 cases per 100000 people (36).

Genetics.-MELAS is considered a prototypic PMD, as affected patients have a maternal pattern of inheritance that is due to pathogenic variants in mtDNA with variable heteroplasmy. With this PMD, a certain level (threshold) of affected mitochondria in different tissues is required before clinical symptoms manifest (37). The first described pathologic variant associated with MELAS is the m.3243A $>\mathrm{G}$ mutation in the MT-TL1 gene, which is responsible for approximately $80 \%$ of 
Table 1: Main Differential Diagnoses of Leigh Syndrome Associated with Signal Intensity Changes in the Basal Ganglia, Thalamus, and/or Brainstem

Disease(s) with Signal Intensity Changes

Viral encephalitides

Demyelinating diseases such as acute disseminated encephalomyelitis, anti-MOG, CNS vasculitis, and pediatric autoimmune demyelination

Acute necrotizing encephalitis of childhood

Glutaric aciduria type 1

L-2-hydroxyglutaric aciduria

Propionic and methylmalonic acidemia

Wernicke encephalopathy

Biotin-thiamine-responsive BG disease

Kernicterus

Hypoxic injury

Carbon monoxide poisoning

Hemolytic-uremic syndrome

Maple syrup urine disease

Wilson disease

Huntington disease

Note. $-\mathrm{BG}=$ basal
Thalami and/or BG signal intensity changes, often with CSF pleocytosis

Signal intensity changes affecting the cerebral WM, thalami, brainstem, and spinal cord, often in an asymmetric fashion

Bilateral symmetrical thalami with or without BG involvement, which may have hemorrhage, cavitation, and contrast enhancement secondary to viral infections such as influenza A and B, parainfluenza, varicella, enterovirus, and human herpesvirus 6; this disease is immune mediated or due to underlying genetic vulnerability caused by pathogenic variants in $R A N B P 2$ gene

Progressive macrocephaly, widening of the sylvian fissures, and BG signal intensity changes, especially in the globi pallidi

Bilateral and symmetric T2-hyperintense changes in the subcortical and periventricular WM, BG, and dentate nuclei

Ketoacidosis, hypotonia, developmental delay, dehydration, seizure, lethargy, and BG signal intensity changes

Typical neuroimaging features include signal intensity changes in the mammillary bodies, dorsomedial thalami, tectal plate, periaqueductal gray matter, and BG

Cortical and WM atrophy, diffuse or patchy cerebral WM signal intensity changes, and Leigh syndrome features such as signal intensity abnormalities in the BG, medial thalami, dorsal midbrain, pons, and medulla, with total or partial resolution after treatment with vitamin B7 (biotin) and vitamin B1 (thiamine); this disease is caused by variants in the $S L C 19 A 3$ gene, which encodes a thiamine transporter

Chronic bilirubin encephalopathy with globus pallidus changes

BG signal intensity changes after a neonatal ischemic insult, near drowning, cardiac arrest, or nonaccidental trauma

BG signal intensity changes, particularly in the globi pallidi

Microangiopathic hemolytic anemia, acute renal failure, thrombocytopenia, and microthrombosis in patients younger than 5 years after diarrhea caused by infection with Shiga toxin-producing Escherichia coli; may show BG signal intensity changes

Characteristic urine odor and diffuse intramyelinic edema, especially of the cerebellarWM, dorsal brainstem, cerebral peduncles, and posterior limb of the internal capsule, globi pallidi, thalami, perirolandic cerebral WM, and dorsal brainstem

Signal intensity changes in the putamina, globus pallidus, caudate nuclei, ventrolateral thalami, and mesencephalon, with characteristic "face of the giant panda" sign

Typically with caudate nuclei head atrophy
MELAS cases (38). The MT-TL1 gene provides instruction for making a transfer RNA (tRNA), designated as tRNA ${ }^{\mathrm{Lu}(\mathrm{UUR})}$, which attaches to the cognate amino acid leucine during protein assembly and inserts this amino acid into appropriate locations in the growing protein (39). The heteroplasmy levels of MELAS-causing mtDNA variants can vary greatly from tissue to tissue in an individual, including but not limited to blood, saliva, urine sediment, skin fibroblasts, and muscle (40). Of note, the m.3243A>G mutation has been noted to decrease in blood at a rate of approximately $1.4 \%$ a year, so testing of multiple tissues may be needed for an accurate understanding of the mutation burden. MELAS variants most commonly affect complexes I and IV, since the number of mtDNA-encoded subunits is highest in these complexes (41). 


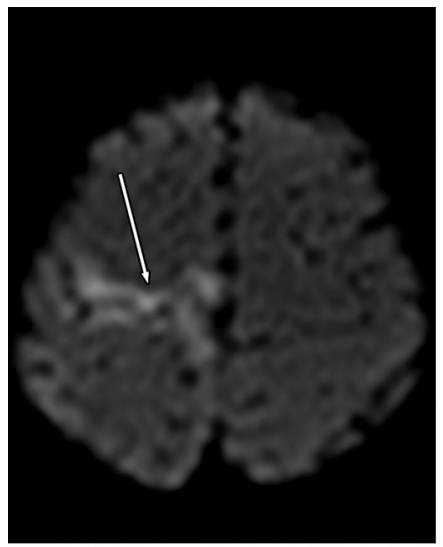

a.

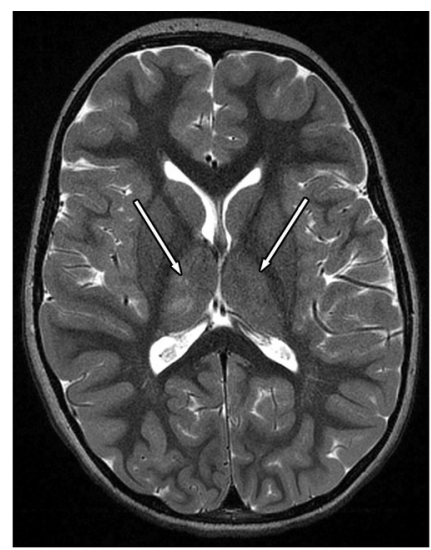

b.

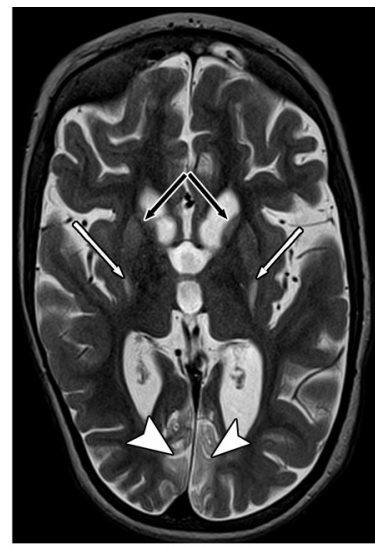

c.

Figure 8. Seizures and encephalopathy due to POLG-RDs in three patients. (a, b) Axial diffusionweighted (a) and T2-weighted (b) MR images show perirolandic restricted diffusion in the right cerebral hemisphere (arrow in a) and bithalamic signal intensity changes (arrows in b). (c) Axial T2-weighted MR image shows bilateral occipital encephalomalacia (arrowheads), bilateral signal intensity changes, and volume loss of the caudate nuclei (black arrows) and putamina (white arrows), in addition to diffuse volume loss.

Clinical Features.-The onset of MELAS typically occurs during childhood in an otherwise healthy individual. The first onset of symptoms frequently occurs in persons between the ages of 2 and 20 years, although it can sometimes be delayed until between 10 and 40 years of age. The most common initial symptoms of MELAS are seizures, recurrent headaches, poor appetite, failure to thrive, muscle weakness, short stature, strokelike episodes, sensorineural hearing loss, exercise intolerance, visual symptoms (cortical vision loss, pigmentary retinopathy, ptosis), developmental delay, and recurrent vomiting, which typically manifest after a stressor such as surgery or infection (42). Other symptoms include gastrointestinal conditions such as dysmotility and pseudo-obstruction, diabetes mellitus, hirsutism, and learning difficulties. More mildly affected maternal relatives may display symptoms such as hearing loss and diabetes (ie, maternally inherited deafness and diabetes) without strokelike episodes (43).

Imaging Findings.-The strokelike episodes are probably caused by abnormal mitochondria in the endothelial and smooth muscle cells of blood vessels, leading to impairment of the autoregulatory vascular mechanisms. These episodes must be differentiated from ischemic vascular strokes because different management is required (44). MELAS lesions are most often (about $90 \%$ of cases) located in the posterior and lateral brain regions (occipital, parietal, or temporal lobes). CT findings are nonspecific and include symmetric calcifications in the basal ganglia, parietal and occipital focal low-attenuation (infarct-like) areas, and generalized atrophy with prominent dilated
Table 2: Diagnostic Criteria for MELAS

Category A: clinical findings of strokelike episodes

Headache with vomiting

Seizure

Hemiplegia

Cortical blindness or hemianopsia

Acute focal lesion observed at brain imaging (focal brain abnormalities at $\mathrm{CT}$ and/or MRI)

Category B: evidence of mitochondrial dysfunction

High lactate levels in plasma and/or CSF, or deficiency of mitochondria-related enzyme activities

Mitochondrial abnormalities at muscle biopsy

Definitive gene mutation related to MELAS

Definitive MELAS

Two features from category A and two features from category B (total of four features or more)

Suspected MELAS

One feature from category $\mathrm{A}$ and two features from category B (total of three or more features)

occipital horns (45). MRI findings are more specific and may include T2-hyperintense migrating ("skipping") lesions located in the cortex preferentially but also in the subcortical white matter. The lesions may affect any part of the brain (including the cerebellum) but predominantly involve the parietal and occipital lobes in nonvascular territories. The basal ganglia may be involved in the form of chronic infarction, necrosis, and calcification (46). During the course of the disease, lesions may resolve with clinical improvement or residual atrophy, while new lesions may appear in other regions with a typical pattern (Fig 9) (46).

In the acute and subacute stages of MELAS, the affected areas show high signal intensity on 


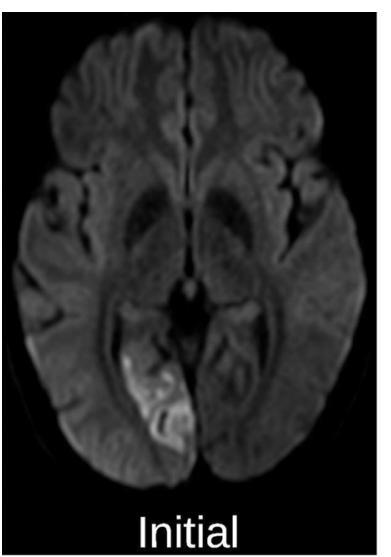

a.

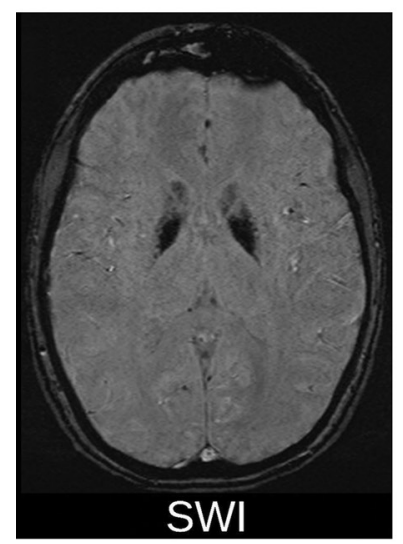

e.

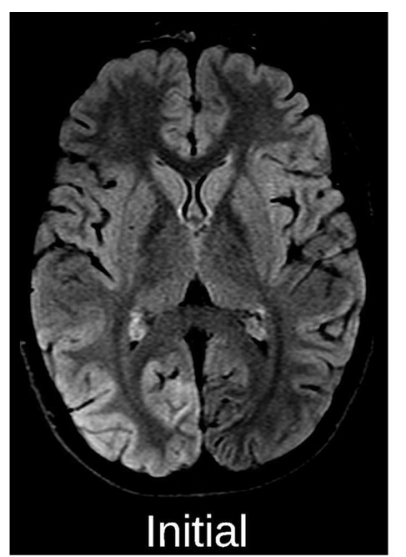

b.

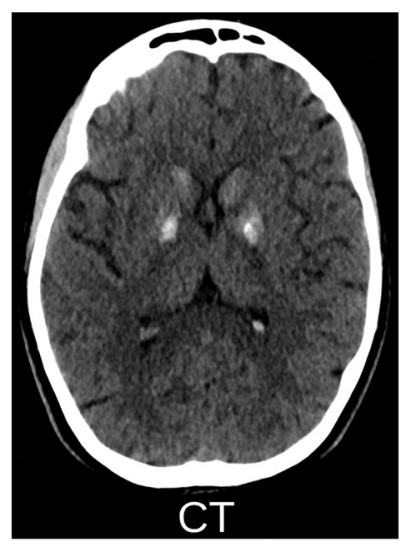

f.

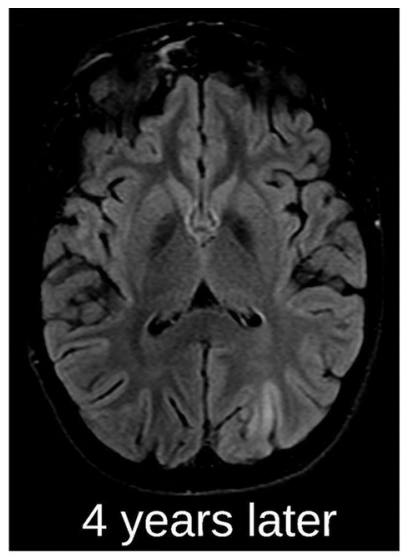

c.

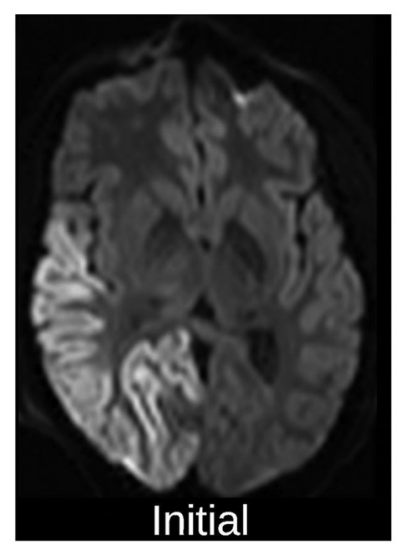

g.

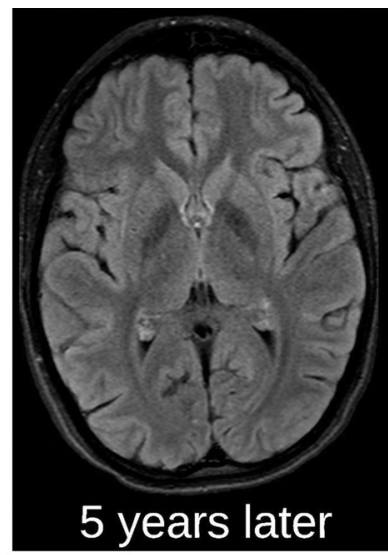

d.

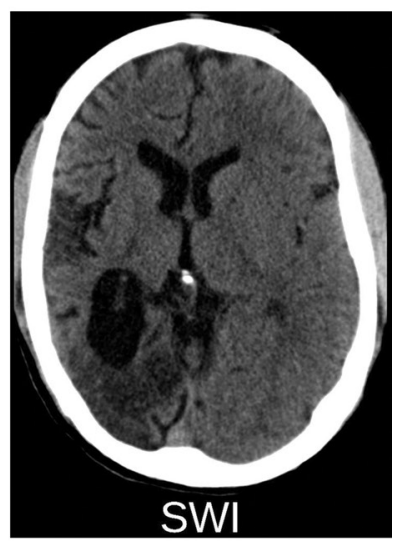

h.

Figure 9. MELAS, with different disease evolution, in an 11-year-old girl with difficulty swallowing, gagging, dysarthria, bilateral sensorineural hearing loss, and strokelike episodes (a-f) and in a 13-year-old girl with a history of headaches and strokelike symptoms (g, h). (a, b) Axial initial diffusion-weighted (a) and FLAIR (b) MR images in the first patient show a small area of restricted diffusion in the right medial occipital lobe (a) and a larger area of signal intensity abnormalities (b) encompassing two arterial territories, of both the middle and posterior cerebral arteries. In $\mathbf{a}$ and $\mathbf{b}$, signal intensity changes are more evident, involving the cortical ribbon. (c) Axial follow-up MR image 4 years later shows that the signal intensity changes in the right cerebral hemisphere are completely resolved. A new focus of signal intensity abnormalities is seen in the left occipital lobe. (d) Subsequent axial follow-up MR image 5 years after the initial study shows complete resolution of the lesion in the left occipital lobe. (e, $f$ ) Axial susceptibilityweighted MR image (e) and CT image (f) show signs of calcium deposits. (g) Axial initial diffusion-weighted image in the second patient shows a large area of restricted diffusion involving the temporal, parietal, and occipital lobes (mainly cortical). (h) Axial follow-up susceptibility-weighted MR image shows that encephalomalacia of the same lesions has developed.

diffusion-weighted MR images and low values on apparent diffusion coefficient (ADC) maps. On the ADC map, lesions may show increased, decreased, or mixed signal intensity, suggesting the coexistence of cytotoxic (low ADC) and vasogenic (high ADC) edema within a strokelike lesion (47). At MR spectroscopy, a lactate peak due to mitochondrial dysfunction and reduction of the $\mathrm{N}$-acetyl aspartate peak, indicating neuronal loss, may be identified in both affected and nonaffected areas and in the CSF (voxels placed over the ventricles) (48). In contrast to the typical hypoperfusion seen with acute vascular stroke, there is no reduction in regional cerebral blood flow in the affected areas in individuals with MELAS, with several studies instead demonstrating normal or even increased perfusion. Cerebral angiography most commonly reveals normal findings, with the most common abnormality being increased caliber of arteries or veins or a capillary blush with early venous filling (49).

Differential Diagnosis.—Cortical changes can be seen in cases of arterial infarction (respecting an arterial territory), neonatal hypoglycemia, posterior reversible encephalopathy syndrome (secondary to arterial hypertension or chemotherapy), hemiplegic migraines (throbbing pain, nausea, and light and/or sound sensitivity), and encephalitis (mostly viral), and following seizures (postictal changes) (50).

\section{Kearns-Sayre Syndrome}

Definition.-Kearns-Sayre syndrome typically consists of the clinical triad of chronic progressive external ophthalmoplegia (including ptosis), 
retinal pigmentary degeneration, and cardiac conduction abnormalities, with onset in persons younger than 20 years (51). The diagnosis can be established by a combination of consistent clinical, radiologic, pathologic, biochemical, and molecular abnormalities (52). Molecular confirmation can be achieved with mtDNA deletion studies in noninvasive tissue (blood, buccal swab, urine) or with muscle biopsy, which may also reveal characteristic ragged red fibers (53). KearnsSayre syndrome has an estimated incidence of one to three cases per 100000 people (54).

Genetics.-Kearns-Sayre syndrome is a multisystemic disorder caused by a single large-scale (1.1-10.0 kilobases) mtDNA deletion, which is typically de novo or sporadic (51). Empiric data suggest that an affected woman has roughly a $4 \%$ chance to pass on an mtDNA deletion to her children. If the mother of an affected child is not affected, the risk to the siblings is estimated to be $1 \%-4 \%$ owing to potential germline mosaicism. The most common deletion, referred to as the common $4977 \mathrm{bp}$ deletion, accounts for more than one-third of cases (55). The mtDNA deletion associated with Kearns-Sayre syndrome results in the loss of genes that are important for mitochondrial protein formation and OXPHOS, causing a decrease in cellular energy production.

Clinical Features. - In addition to the clinical triad, patients with Kearns-Sayre syndrome may be found to have cognitive decline or dementia, sensorineural hearing loss, cardiomyopathy, myopathy associated with limb weakness, and endocrine dysfunction (diabetes mellitus, hypoparathyroidism, hypogonadotropic hypogonadism, adrenal insufficiency, and growth hormone deficiency). These patients may also have short stature, failure to thrive, chronic renal failure, cerebellar ataxia, and elevated CSF protein $(>100 \mathrm{mg} / \mathrm{dL})(52)$.

Imaging Findings.-The neuroimaging findings of Kearns-Sayre syndrome are variable, ranging from normal to lesions in the subcortical and deep white matter, corpus callosum, basal ganglia, thalami, dorsal brainstem, ventral brainstem, superior and middle cerebellar peduncles, cerebellar white matter, and spinal cord. Typical neuroimaging findings include T2-hyperintense lesions involving the subcortical white matter (subcortical arcuate fibers), with sparing of the periventricular white matter, at MRI. Early involvement of the subcortical white matter is a typical neuroimaging feature of Kearns-Sayre syndrome that may help in differentiating this syndrome from other entities such as lysosomal and peroxisomal disorders, in which the subcortical white matter is spared until the late stage of the disease (56).

Involvement of the mediodorsal thalamus, basal ganglia (more commonly the globus pallidus and caudate nucleus), and brainstem tegmentum also is commonly seen (56). In the acute stage, lesions may show restricted diffusion (Fig 10). CT or MRI may show calcified deposits in the basal ganglia in the late stages. An unusual pattern of radially oriented T2 lowsignal-intensity stripes within the abnormally myelinated white matter also has been described (57). Cerebellar atrophy is commonly observed in the later stages of the disorder (58).

Differential Diagnosis.—Common causes of basal ganglia calcification include hypothyroidism, hyperparathyroidism, hypoparathyroidism, pseudohypoparathyroidism (associated with endocrinologic abnormalities), AicardiGoutières syndrome (microcephaly, progressive encephalopathy, bilateral calcification of the basal ganglia, spastic quadriplegia), and familial idiopathic basal ganglia calcification type 1 or Fahr disease (not associated with endocrinologic abnormalities) (59). Other PMDs, such as MELAS and Leigh syndrome, may show basal ganglia calcification. The most relevant causes of leukoencephalopathy are metachromatic leukodystrophy (frontal predominance) L-2-hydroxyglutaric aciduria, Krabbe disease (parieto-occipital predominance, and optic nerve and chiasm thickening), and Alexander disease (frontal predominance) (60).

\section{Leber Hereditary Optic Neuropathy}

Definition.-In LHON, the optic nerve retinal ganglion cells are the target, resulting in retinal ganglion cell degeneration, optic nerve atrophy, and central vision loss. LHON traditionally has been believed to be an organ-specific disease, being the most common of the inherited optic neuropathies. However, "LHON-plus" phenotypes may include additional extraocular features (Fig $11)$. The incidence of $L H O N$ ranges from one in 30000 to one in 50000 persons (61).

Genetics.-LHON follows a maternal mitochondrial DNA pattern of inheritance with reduced penetrance; approximately $50 \%$ of males with mutation and approximately $10 \%$ of females with mutation are affected. More than $90 \%$ of LHON cases are the result of one of three $\mathrm{mtDNA}$ point pathogenic variants in the mtDNA genes, m.11778>A, m.3460G $>\mathrm{A}$, or m.14484T $>C$, all of which encode the complex 


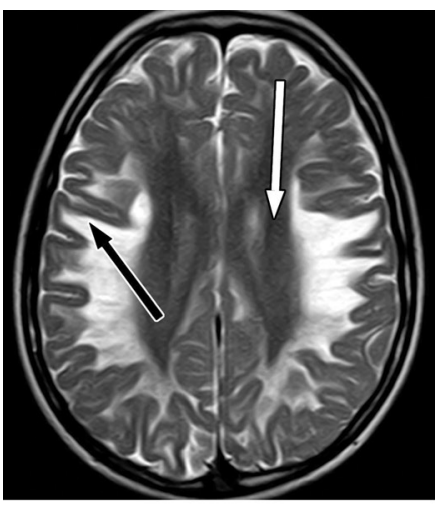

a.

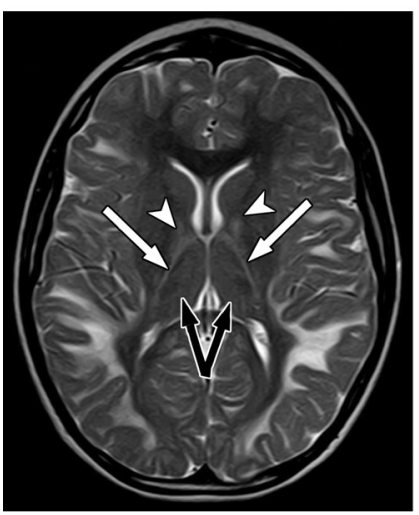

d.

Figure 10. Headache, dizziness, sensorineural hearing loss, and bilateral leg weakness in a 13-year-old boy with Kearns-Sayre syndrome. (a) Axial T2-weighted MR image shows diffuse hyperintensity involving the bilateral white matter, the arcuate fibers (black arrow) in particular, but sparing the periventricular white matter (white arrow). (b) Axial diffusion-weighted MR image shows diffuse increased signal intensity (arrow), which manifested on the corresponding ADC map (not shown) as areas of low and high signal intensity, consistent with cytotoxic edema associated with areas of vasogenic edema. (c-f) Coronal (c) and axial (d-f) T2-weighted MR images show bilateral involvement of the superior temporal gyri and Heschl gyri (arrows in c), increased T2 signal intensity involving the globi pallidi (arrowheads in d) and posterior limbs of the internal capsule (white arrows in d) and thalami (black arrows in d), and diffuse signal intensity abnormalities in the midbrain (arrow in e) and pontine tegmentum (arrow in $\mathrm{f}$ ), associated with mild widening of the sulci surrounding the cerebellar folia.

I subunit of the respiratory chain (62). In the majority of cases, these mutations are homoplas$\operatorname{mic}(62)$.

Clinical Features._-LHON usually manifests as painless, subacute, and central visual loss. It frequently manifests in individuals aged $20-40$ years and more frequently in males. Nevertheless, individuals of either sex and from all age groups can develop LHON. In the majority of cases, visual impairment eventually becomes bilateral (documented in up to $99.4 \%$ of cases), with about $50.3 \%$ of all patients demonstrating sequential onset. In the latter group, the median inter-eye delay has been reported as 12.8 weeks (63). Hence, a patient who has had unilateral optic neuropathy for longer than 1 year is highly unlikely to suffer from LHON-related vision loss (64). Unaffected carriers may become symptomatic once they are exposed to specific triggers, such as cigarette smoke, alcohol, or specific antibiotics (ie, mac- rolides, aminoglycosides, ethambutol, isoniazid, and linezolid) (65). Extraocular symptoms that can be seen in conjunction with LHON-related mutations, causing the "LHON plus" phenotype, include tremor, peripheral neuropathy, movement disorders, multiple sclerosis-like illness, myopathy, and cardiac conduction abnormalities.

Imaging Findings.-The diagnosis of $\mathrm{LHON}$ is usually based on clinical findings, especially if classic ophthalmologic features are present and a clear maternal history is elicited (66). Molecular genetic testing still remains the reference standard for confirming that the patient harbors one of the three primary mtDNA LHON pathologic variants. CT and MRI findings are usually normal in patients with LHON, but there are reports of nonenhancing T2 hyperintensity in the optic nerve and sheath distention, secondary to slight edema, gliosis, or optic nerve atrophy (67). 
Figure 11. Maternal and genetically proven history of LHON plus, and long-standing history of hypotonia, optic nerve hypoplasia, asymmetric gait, and leg weakness in a 5-year-old boy. (a-d) Coronal (a) and axial (b, c) T2-weighted, and axial FLAIR (d) MR images show bilateral optic nerve thinning (arrows in a), bilateral posterior putaminal T2-hyperintense foci (arrows in b), bilateral midbrain T2 hyperintensity in the oculomotor nuclei (arrow in c), and bilateral midbrain T2 hyperintensity in the lateral substantia nigra (arrow in d). (e, f) MR spectroscopy revealed an abnormal lactate peak at $1.33 \mathrm{ppm}$ (arrow in e) with a short echo time (30 msec) and an inverted small doublet peak at $1.33 \mathrm{ppm}$ (arrow in $\mathrm{f}$ ) with an intermediate echo time (135 $\mathrm{msec}$ ).

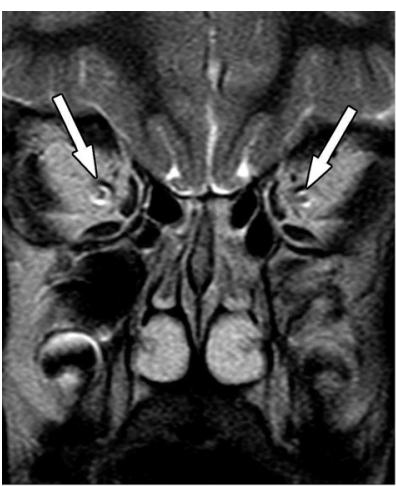

a.

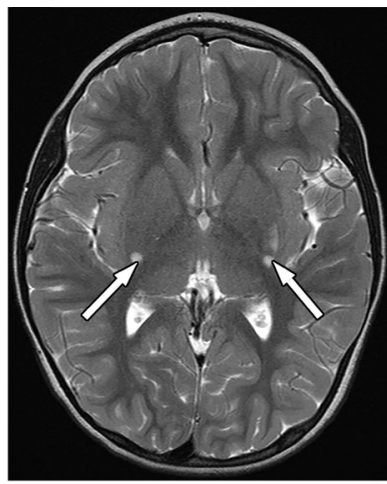

b.

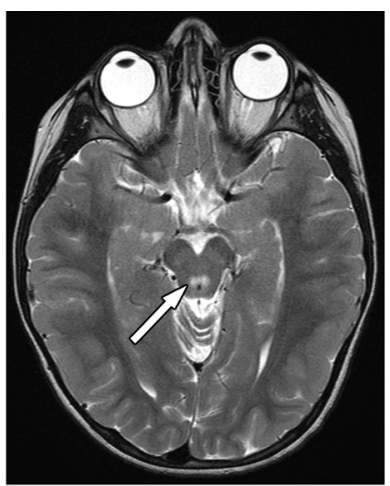

c.

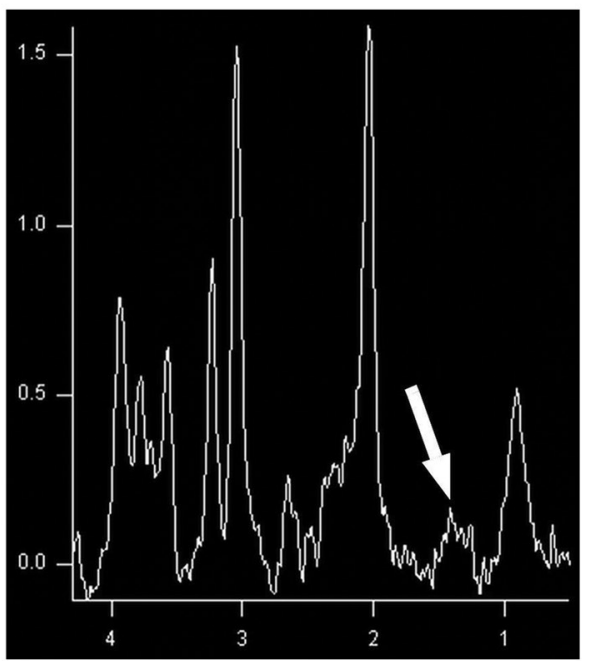

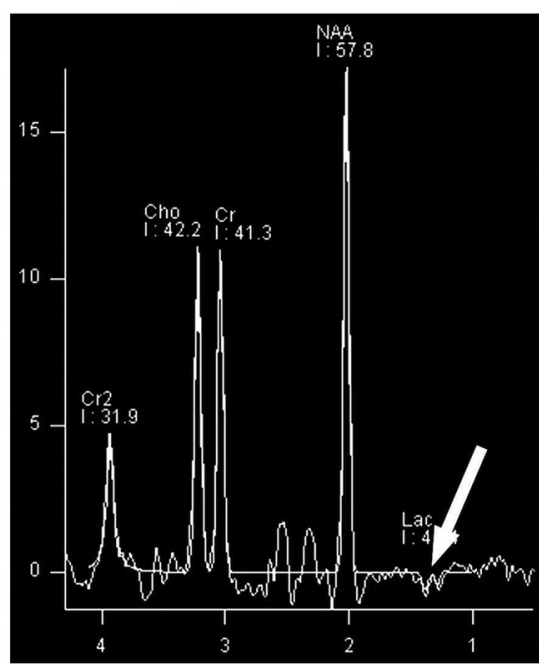

f.

.

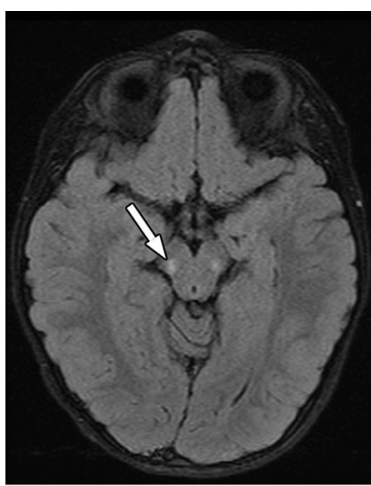

d.

e.

Differential Diagnosis.-Bilateral optic nerve atrophy can be seen (rarely as an isolated finding) with several conditions such as hypoxic-ischemic injury and hydrocephalus. It can also be seen during the chronic stages of demyelinating diseases such as neuromyelitis optica and multiple sclerosis (68).

\section{PDH Complex Deficiency}

Definition.-The PDH complex is formed by three nuclear-encoded enzymes on the inner mitochondrial membrane that catalyze the oxidative decarboxylation of pyruvate into the formation of acetyl coenzyme A, carbon dioxide, and nicotinamide adenine dinucleotide plus hydrogen (69). Genetic variants in the PDH complex make up one of the most common causes of primary lactic acidosis and elevated pyruvate levels in children. PDH complex deficiency can manifest clinically as (a) neonatal encepha- lopathy with lactic acidosis, (b) nonprogressive infantile encephalopathy, (c) Leigh syndrome, or (d) relapsing ataxia (70). The incidence and prevalence of $\mathrm{PDH}$ remain unknown.

Genetics.-PDH complex deficiency is genetically heterogeneous with variable inheritance patterns. It is commonly caused by variants in the gene encoding the E1- $\alpha$ polypeptide (PDHA1), which maps to chromosome Xp22 (69). Other genetic causes of PDH complex deficiency include those related to $D L A T, D L D$, LIAS, MPC1, PDHB, PDHX, and PDP1, all of which are inherited in an autosomal recessive manner.

Clinical Features.-The signs and symptoms of PDH can start at any time between a person's birth and late childhood, but more commonly in infancy. The clinical phenotypes differ widely. Patients with neonatal PDH complex deficiency 


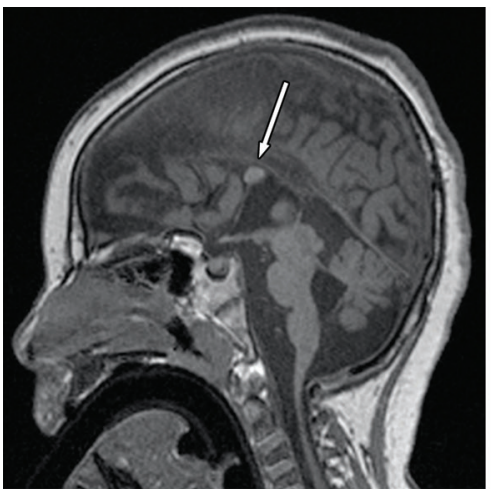

a.

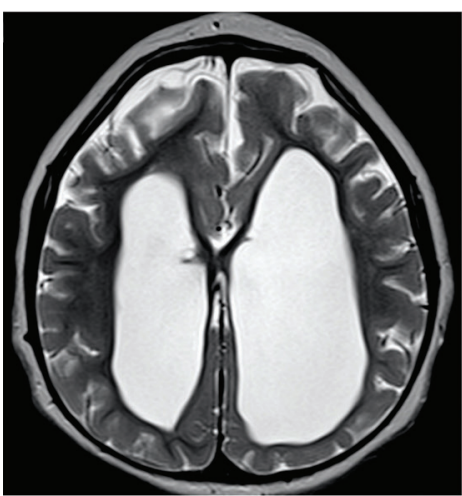

d.

Figure 12. PDH deficiency in a 4-year-old boy with microcephaly, developmental arrest, apneic episodes, and hypothermia. (a-e) Sagittal (a), axial (b), and coronal (e) T1-weighted, and axial T2-weighted (c, d) MR images show almost complete absence of the corpus callosum (arrow in $\mathbf{a}$ ), indistinct basal ganglia (arrow in $\mathbf{b}$ and $\mathbf{c}$ ), ventriculomegaly (d), and bilateral subependymal cysts (arrows in e). (f) Axial diffusion-tensor image shows a remnant of the commissural white matter of the dysgenetic corpus callosum (arrow).

have marked congenital lactic acidosis and encephalopathy. There may be a genetic predisposition for females in this presentation, which is classically characterized by early clinical onset and structural brain abnormalities (agenesis of the corpus callosum and intraventricular cysts) and may be fatal in males (71). Leigh syndrome due to PDH complex deficiency manifests clinically with seizures, dystonia, microcephaly, cerebral atrophy, basal ganglia or brainstem lesions, dysgenesis of the corpus callosum, and elevated lactate and pyruvate levels (leading to a low serum lactate-pyruvate ratio), eventually resulting in precocious death. In milder forms, patients may have episodic ataxia syndrome triggered by carbohydrate ingestion (72).

Imaging Findings.-Brain abnormalities may be due to developmental (perinatal) anomalies or neurodegenerative changes. Perinatal findings of PDH complex deficiency include asymmetric supratentorial ventriculomegaly, ventricular septa, parenchymal or subependymal pseudocysts, severe white matter volume loss, agenesis or dysgenesis of the corpus callosum, hypoplasia of the pons and medulla, and migrational anomalies, including periventricular heterotopia, polymicrogyria, and pachygyria. In milder forms of the disease, MRI of the brain may show signal intensity changes in the basal ganglia that range from small punctate lesions to the full pattern of Leigh syndrome with bilateral lesions of the basal ganglia, brainstem, and dentate nuclei. In the long term, the disease can result in brain atrophy (Fig 12). MR spectroscopy may help diagnose PDH complex deficiency by revealing elevated lactate levels, aiding in the differential diagnosis (73).

\section{CoQ10 Deficiency}

Definition.-CoQ10 is a lipophilic molecule that has redox capacity and is located in cell membranes. CoQ10 is an endogenously synthesized essential cofactor of the mitochondrial electron transport chain. It is responsible for carrying electrons from complexes I and II, and the oxidation of fatty acids and branched-chain amino acids via flavin-linked dehydrogenases, to complex III (74). CoQ10 deficiency has been associated with a large number of different clinical phenotypes, which can be further divided into primary forms (due to variants in CoQ10 genes) and 


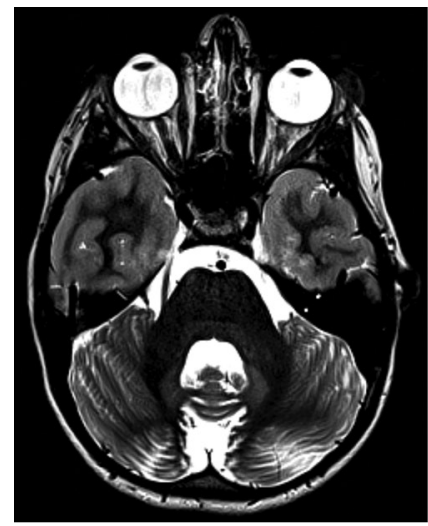

a.

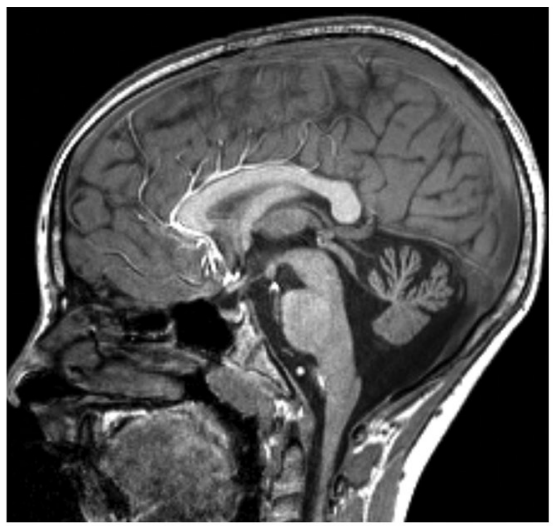

b.

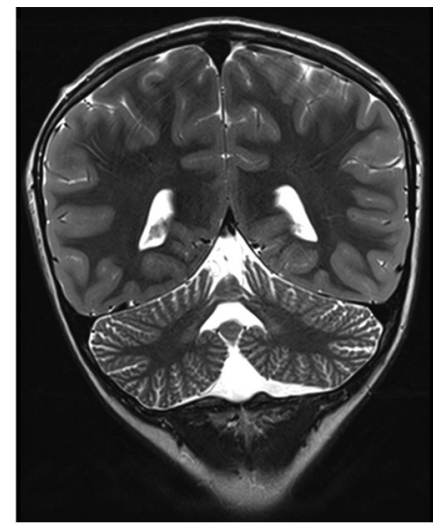

c.

Figure 13. CoQ10 deficiency causing hypotonia and ataxia in a 9-year-old boy. Axial (a) and coronal (c) T2-weighted, and sagittal T1-weighted (b) MR images show volume loss with widening cerebrospinal fluid around the cerebellar folia.

secondary forms (defects in genes not directly involved in CoQ10 biosynthesis or defects due to nongenetic factors such as statin use for hypercholesterolemia) (75). The incidence and prevalence of CoQ10 deficiency remain unknown.

Genetics.-At least nine genes from the nuclear genome are involved in CoQ10 biosynthesis (75). CoQ10 deficiency is inherited in an autosomal recessive pattern.

Clinical Features.-The age at onset and the disease severity in persons with primary CoQ10 deficiency are variable. In the most severely affected individuals, the disease manifests during childhood and results in significant neurologic findings, including nystagmus, intellectual disability, seizures, dystonia, hypotonia, spasticity, cerebellar ataxia or pyramidal dysfunction, and peripheral neuropathy. Other comorbidities include optic atrophy, cataracts, sensorineural hearing loss, hypertrophic cardiomyopathy, muscle weakness, and nephropathy with focalsegmental glomerulosclerosis. Six significant phenotypes are described in association with CoQ10 deficiency: (a) a severe multisystem childhood form involving encephalopathy, ataxia, and seizures; (b) cardiomyopathy and renal failure; (c) a cerebellar form with ataxia and cerebellar atrophy; (d) Leigh syndrome; (e) an isolated myopathic form; and $(f)$ steroidresistant nephrotic syndrome (76).

Imaging Findings.-MRI can depict a broad spectrum of abnormalities ranging from completely unremarkable examination findings to Leigh syndrome features. Involvement of the cerebellum, commonly characterized by cerebellar atrophy, is typically observed with CoQ10 deficiency (Fig 13) (77) and associated with T2 cortical hyperintensity, known as a bright cerebellum.
Differential Diagnosis.-Cerebellar atrophy (pure or associated with cerebellar cortical signal intensity changes) is not a specific imaging pattern. Cerebellar atrophy without overt cerebellar signal intensity changes has been described in other PMDs such as PEHO (progressive encephalopathy with edema, hypsarrhythmia, and optic atrophy) syndrome and in several entities such as ataxia telangiectasia, late-onset GM2 gangliosidosis, ataxia-oculomotor apraxia types 1 and 2, mevalonate kinase deficiency, and mutations in the following genes: CACNA1A, SPTBN2,TPP1, ANO10, GRM1, SPTBN2, ITPR1, and GRID2 (78).

Cerebellar atrophy associated with cerebellar cortex signal changes may be seen in the later stages of other PMDs and also has been described in cases of infantile neuroaxonal dystrophy, Marinesco-Sjögren syndrome, congenital disorders of glycosylation 1a, Christianson syndrome, late infantile neuronal ceroid lipofuscinosis, pontocerebellar hypoplasia type 7 , and some forms of nonprogressive cerebellar ataxia (78).

\section{Leukoencephalopathy with Brainstem and Spinal Cord Involvement and Lactate Elevation}

Definition.-LBSL was initially described in 2003 on the basis of the characteristic symptoms of slowly progressive cerebellar ataxia, spasticity, and dorsal column dysfunction, sometimes with mild cognitive deficit or decline. LBSL is a rare autosomal recessive disease with an onset typically during childhood; about $50 \%$ of cases manifest in persons younger than 6 years. Nevertheless, later onset can occur during adolescence and adulthood, mainly in females (79). The incidence and prevalence of LBSL are unknown.

Genetics.-LBSL can be caused by homozygous or compound heterozygous variants in the 


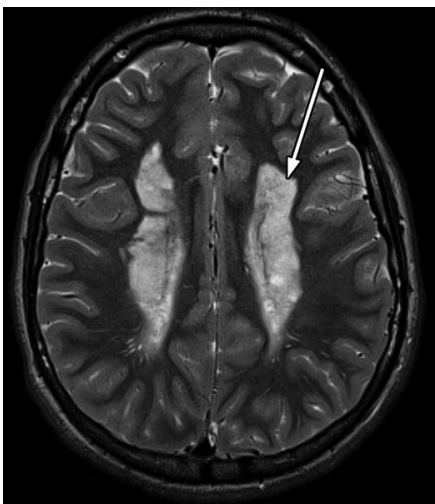

a.

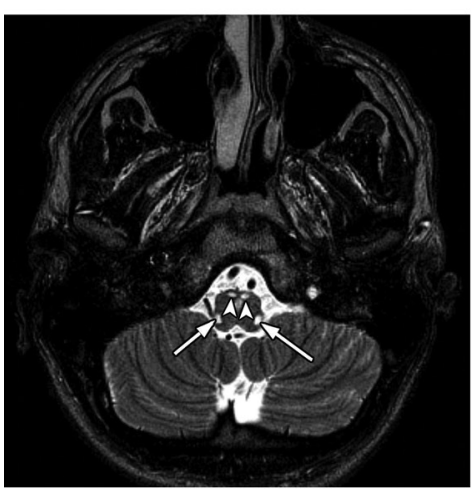

d.

Figure 14. LBSL in a 12-year-old boy with ataxia and mild developmental delay. Axial T2-weighted MR images show diffuse white matter hyperintensity with sparing of the subcortical white matter (arrow in a) and signal intensity changes in the intraparenchymal course of the trigeminal nerves (arrow in b), the inferior cerebellar peduncles (arrow in c), the lateral corticospinal tracts (arrows in $\mathbf{d}$ ), a pyramidal tract at the level of the medulla oblongata (arrowheads in $\mathbf{d}$ ), the decussating medial lemniscus (arrow in e), and the lateral corticospinal tracts and dorsal columns of the spinal cord (f).

nuclear gene that encodes mitochondrial aspartyl-tRNA synthetase (DARS2) on chromosome $1 \mathrm{q} 25$ (79). The DARS2 gene is part of the family of mitochondrial aminoacyl-tRNA synthetase (mt-aaRS) genes that code for enzymes with a key role in mitochondrial protein synthesis. These are critical enzymes in mitochondrial translation, as they catalyze the specific attachment (conjugation) of a cognate amino acid-indicated by the first letter in the mt-aaRS (ie, mt-AspRS = $D A R S 2)$ - to its mt-tRNA (80).

Clinical Features.-The clinical manifestations of LBSL are variable. Persons with infantile onset of the disease are more severely affected than are other affected individuals, with rapid disease progression and a higher risk of death. However, in general, patients with LBSL can have a long life span. Clinically, there is a slow progression of cerebellar ataxia. Most patients require some assistance with walking after 18 years of age but are not fully wheelchair dependent until their 2 nd or 3 rd decade of life (81). These patients exhibit less severe motor disability. Within the LBSL patient cohort, other reported symptoms include mild speech delay, learning problems, visuospatial defects, and intellectual disability or cognitive decline.

Imaging Findings. - The main neuroimaging findings of LBSL were described more than a decade ago, but additional diagnostic criteria have been published (82). To fulfill the MRI criteria for this diagnosis, there should be at least abnormal signal intensity in all of the following brain structures: (a) the cerebral white matter (with relative subcortical sparing); (b) the decussating medial lemniscus or pyramidal tract at the level of the medulla oblongata, or both; and (c) the lateral corticospinal tracts and dorsal columns of the spinal cord. In addition, the minor sign of signal intensity abnormalities in one of the following regions should be present: (a) cerebellar white matter, (b) superior and inferior cerebellar peduncles, (c) anterior spinocerebellar tracts in the medulla oblongata, (d) trigeminal tracts in the mesencephalon, (e) intraparenchymal part of the trigeminal nerve, $(f)$ posterior limb of the internal capsule, and (g) splenium of the corpus callosum (Fig 14) (82). Diffusion-weighted imaging may depict scattered areas of restricted diffusion in the 
white matter, a common feature in mitochondrial leukoencephalopathies that likely reflects chronic myelin splitting and intramyelinic vacuole formation (83).

\section{Clues to Diagnosis and Roles of Imaging}

The diagnosis of PMD can be challenging. These are rare disorders with clinical and imaging manifestations that overlap considerably with many other disorders. However, some clinical clues may hint at the presence of a PMD. Multisystem dysfunction can be a clue, particularly when it involves the CNS, striated muscles, heart, and liver. For example, developmental regression and neurologic symptoms seen at chest radiography in a child who has a pacemaker owing to heart blockage may be an anecdotal clue to suspected mitochondrial disease such as Kearns-Sayre syndrome. Unexplained progressive visual loss, ophthalmoplegia, ptosis, and unexplained sensorineural hearing loss, particularly in combination with other symptoms, may be other clinical markers of PMDs. A family history of PMDs may be helpful, although it may not be present in many cases. Laboratory test abnormalities such as elevated lactate, pyruvate, carnitine, and alanine levels may be additional clues to mitochondrial dysfunction. Imaging has an important role in the evaluation of CNS manifestations of PMDs. In the following sections, we review the various neuroimaging features of these disorders.

\section{US Features and Roles}

Neurologic US may be useful in the setting of a few PMDs that manifest with primary lactic acidosis, PDH complex deficiency and pyruvate carboxylase deficiency in particular. Both of these disorders involve subependymal cysts and mitochondrial complex IV (cytochrome c oxidase) deficiency (84). In addition to subependymal cysts, PDH complex deficiency may also involve ventriculomegaly accompanied by a gyration or corpus callosum abnormality (agenesis or dysgenesis) (85), diffuse swelling, and increased echogenicity of the deep gray and white matter (84). Patients with pyruvate carboxylase deficiency may have ventriculomegaly, especially of the frontal horns, associated with subependymal and periventricular cysts (85), although these cysts are also commonly seen in patients without PMDs. Patients with cytochrome $\mathrm{c}$ oxidase deficiency may have corpus callosum agenesis, ventriculomegaly, reduced gyration, and increased hyperechogenic foci, which may indicate calcification (86).

\section{CT Features and Roles}

CT has limited value in the management of patients with PMD, with the exception that it occasionally depicts calcifications (87). CTdepicted calcifications related to PMDs are seen more commonly in the basal ganglia and cerebellum and more frequently in association with MELAS, Leigh syndrome, Cockayne syndrome (recently linked to mitochondrial dysfunction), Kearns-Sayre syndrome, and other PMDs involving long-term disease (46). However, MRI is much more effective for depicting additional parenchymal changes.

\section{MRI Features and Roles}

MRI is the imaging modality of choice for evaluation of CNS changes in patients with PMDs. There are no pathognomonic MRI findings of PMDs. Conventional MRI features are variable, ranging from highly specific findings to nonspecific findings and normal imaging results, particularly in cases of pure mitochondrial myopathy (87).

The spectrum of MR neuroimaging findings of PMDs includes basal ganglia signal intensity changes, basal ganglia calcification, cortical signal intensity changes and malformations, subependymal cysts, and white matter changes (leukoencephalopathy, white matter cavitation, callosal agenesis or dysgenesis, and delayed myelination or hypomyelination). Other neuroimaging findings include signal intensity changes in the thalamus and subthalamic nucleus, dentate nucleus, pons and pontine tegmentum, and medulla (including the inferior olivary and hypoglossal nuclei). Optic nerve thinning, cerebellar changes (atrophy and signal intensity changes), midbrain changes (signal intensity changes in the midbrain, periaqueductal gray matter, midbrain tegmentum, and substantia nigra), and spinal cord changes (signal changes and cauda equina root enhancement) are other findings. The difficulty lies in the fact that almost all of these and additional imaging findings can be seen in patients without PMD. Therefore, the clinical setting and correlations are important. The locations of the most common PMD lesions are summarized in Table E2.

\section{Lesions in the Basal Ganglia, Thalamus, and Brainstem}

Basal ganglia lesions are very common in PMDs. The most common PMDs associated with basal ganglia abnormalities are Leigh syndrome and POLG-RDs (88). Certain MRI findings, such as bilateral and symmetric focal T2 hyperintensities in the basal ganglia (striatum), thalamus, substantia nigra, and brainstem nuclei at various levels on T2-weighted MR images, can be highly suggestive of PMDs, particularly Leigh syndrome, especially if there is no history of hypoxia, ischemia, or infection. Lesions involving the 
medulla (the inferior olivary nucleus in particular), pons (including the pontine tegmentum), midbrain (particularly the periaqueductal area), cerebellum, and subthalamic nuclei also can be seen in Leigh syndrome secondary to a SURF1 pathogenic variant (89).

Bilateral thalamic and basal ganglia changes associated with cerebral and cerebellar atrophy may also be seen in patients with Kearns-Sayre syndrome. These patients may also have changes in the brainstem tegmentum, cerebral white matter (occasionally with a tigroid appearance), and cerebellar cortex. In addition, white matter involvement is typically subcortical, sparing the periventricular regions. Other diseases associated with thalamic changes include $P O L G-\mathrm{RD}$ and Leigh syndrome.

\section{Cortical Changes}

Cortical changes with a strokelike pattern are the hallmark features of MELAS. In addition, MELAS lesions may manifest with a waxing and waning pattern and as "skipping" lesions that are not restricted to a specific vascular territory. Cortical changes may also occur in cases of POLG-RDs, frequently involving the perirolandic region and occipital lobes (34), and Leigh syndrome (90).

\section{Delayed Myelination and Hypomyelination}

Delayed myelination is a common imaging finding of PMDs. Therefore, its presence within the clinical context of possible PMD requires further workup (87). True hypomyelination can be seen in some diseases such as Cockayne syndrome; in some diseases associated with variants in the tRNA synthetase genes, such as hypomyelination with brainstem and spinal cord involvement and leg spasticity; and in alanyl-tRNA synthetase deficiency (91).

\section{Leukoencephalopathy}

In addition to white matter cavitation, white matter signal intensity changes with a pattern similar to that of leukodystrophy can be seen with several PMDs. The most common PMDs associated with white matter signal intensity abnormalities are Kearns-Sayre syndrome, Pearson syndrome, MELAS, MERRF, and aminoacyl-tRNA synthetase mutations, including LBSL (92).

\section{Cerebral Edema}

Cerebral edema is characterized by the abnormal accumulation of fluid in the brain, which may result from failure of ATP-dependent transporters such as the sodium-potassium pump or from excessive neurotransmitter stimulation (cytotoxic edema) and enhanced vascular permeability (vasogenic edema). Diffusion-weighted imaging is highly sensitive to microstructural abnormalities that are invisible on conventional MR images, particularly in the cerebral white matter. Diffusion-weighted imaging can be the first and sole positive MRI sequence in some cases of PMDs, such as MELAS, and it may be used to diagnose and monitor the temporal evolution of strokelike lesions. Acute strokelike lesions may show a mixed pattern, with cytotoxic edema (low ADC values) in the affected cortex in association with vasogenic edema (high ADC values) in the subjacent subcortical white matter.

In contrast to the typical hypoperfusion seen with acute vascular stroke, there is no reduction in regional cerebral blood flow in the affected areas with MELAS. Rather, several studies have demonstrated normal or even increased perfusion (93). This pattern can also be seen in cases of POLG-RD. Restricted diffusion is commonly found with PMDs, especially when the lesions are in the acute phase, as in MELAS, Leigh syndrome, $P O L G-\mathrm{RD}$, Kearns-Sayre syndrome, and cavitating PMDs in particular, and in tRNA synthetase disorders such as LBSL.

\section{Cavitation}

Cavitating leukoencephalopathy following white matter degeneration is suggestive but not pathognomonic of PMDs. It is more commonly associated with pathogenic variants of the following genes: MERRF, NFU1, NDUFS1, NDUFAF3, APOPT1, LYRM7, NUBPL, IBA57, RMND1, and BOLA-3 (Fig 15) (94).

\section{Calcification}

The most common causes of intracranial calcification in PMDs are MELAS, Leigh syndrome, Cockayne syndrome, Kearns-Sayre syndrome, and long-standing PMDs (Fig 16). The frequency of intracranial calcifications associated with PMDs varies according to the patient cohort and the type of mitochondrial disease, and little is known regarding the overall incidence or prevalence in large cohorts. The incidence of calcification varies according to the type of mitochondrial disease. In small cohorts, the incidence of intracranial calcification may reach up to $54 \%$ of patients with MELAS (95). Intracranial calcification in PMDs is variable, and it may occur in isolation or accompany other parenchymal changes. It may be uni- or bilateral, and symmetric or asymmetric.

Calcifications are more common in the basal ganglia, particularly the globus pallidus, but they may also be present in the caudate nucleus, lentiform nucleus, thalamus, and cerebellum. Recently, a specific entity that manifests with leukoencephalopathy with brainstem and spinal 
Figure 15. Bilateral cavitating leukoencephalopathy in a 17-month-old boy with a pathogenic variant in NDUFV1. (a) Coronal T2-weighted MR image shows diffuse white matter T2 hyperintensity associated with bilateral areas of higher T2 signal intensity (cystic like) (arrows) in the parietal regions. (b) Axial diffusion-weighted image shows the areas of higher T2 signal intensity seen in a with a hypointense signal and surrounded by linear restricted diffusion (arrow). (c, d) On axial (c) and coronal (d) contrast material-enhanced T1-weighted MR images, the cystic areas are nonenhancing, whereas the areas with restricted diffusion show thin peripheral enhancement (arrow).

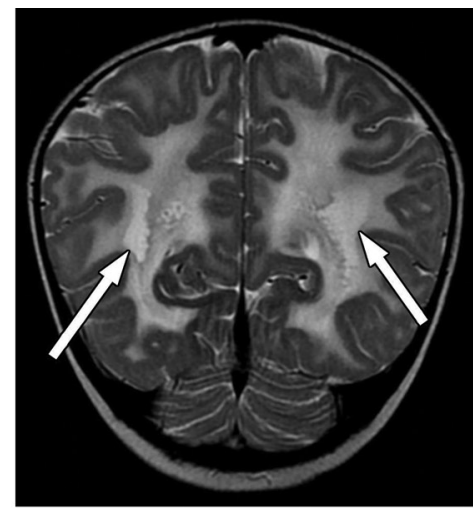

a.
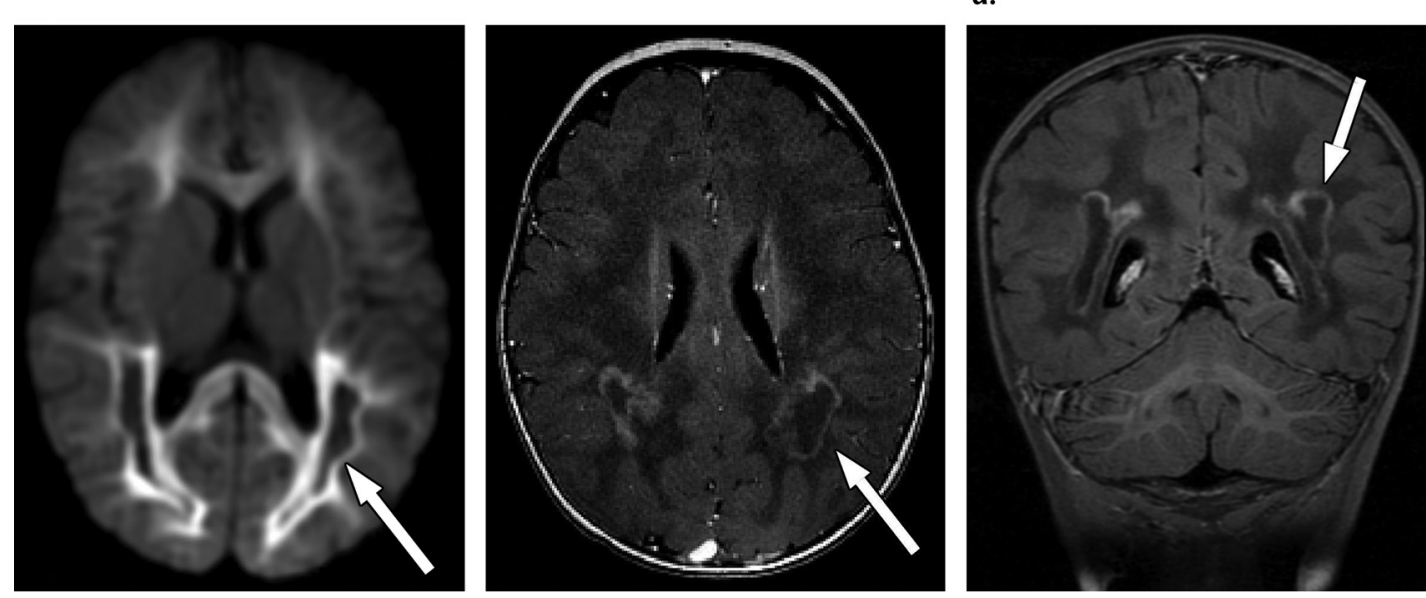

b.

c.

d.

cord calcification has been identified and linked to variants in the KARS gene, one of the tRNA synthetases (96).

\section{Subependymal Cysts}

Prenatal or postnatal MRI or neurologic US findings of subependymal cysts can be seen in cases of PDH complex deficiency and pyruvate carboxylase deficiency, particularly when these cysts are associated with corpus callosum agenesis or dysgenesis. Additional findings include ventriculomegaly, rudimentary cortex, cortical development malformation, and clastic changes (97).

\section{Callosal Agenesis or Dysgenesis}

Several PMDs are associated with agenesis or dysgenesis of the corpus callosum, the following disorders in particular: PDH complex deficiency, pyruvate carboxylase deficiency, aconitase deficiency, fumarase deficiency, complex I assembly defect, and mitochondrial translation defects. Many PMDs are also associated with variants in MRPS16, EARS2, SLC25A1, SLC25A19 (98).

\section{Cerebral and Cerebellar Atrophy}

Cerebral atrophy is a common and nonspecific feature of several PMDs, especially those in the later disease stages (99). Cerebral atrophy may be explained by chronic mitochondrial functional impairment that leads to electron transport chain complex deficiencies (which result in decreased ATP production) and eventually programmed cell death (apoptosis) (100). Cerebral atrophy may progress slowly in some individuals, or it can be rapidly progressive, such as in patients with $P O L G$-RD (34). Focal or global cerebral atrophy may be a sequela of strokelike episodes.

Cerebellar atrophy is also a common feature of multiple PMDs and has been described in association with MELAS, myoclonic epilepsy with ragged-red fibers, Leigh syndrome, maternally inherited diabetes-deafness syndrome, and NARP (neuropathy, ataxia, and retinitis pigmentosa). Cerebellar atrophy has also been linked to chronic progressive external ophthalmoplegia (CPEO) and CPEO plus, POLG-RD, KearnsSayre syndrome, LHON, infantile-onset spinocerebellar ataxia, pontocerebellar hypoplasia type 6 , autosomal dominant optic atrophy, diabetes insipidus, diabetes mellitus, optic atrophy and deafness (ie, Wolfram syndrome), and primary CoQ10 deficiency (101). Neuropathologic studies (102) have shown cerebellar involvement in PMDs based on the presence of cerebellar atrophy, Purkinje cell loss, abnormal dendritic arborization, and strokelike lesions. 


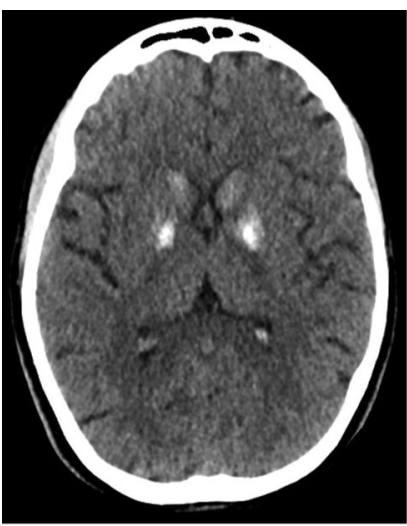

a.

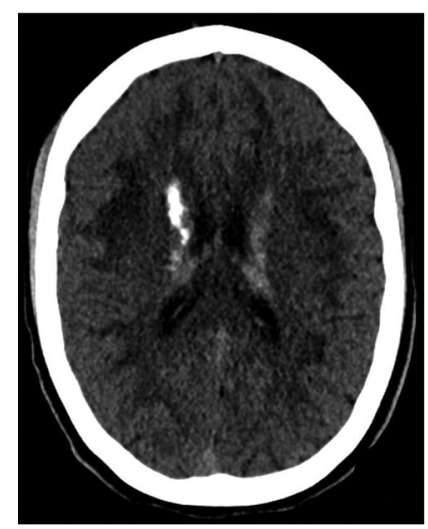

d.

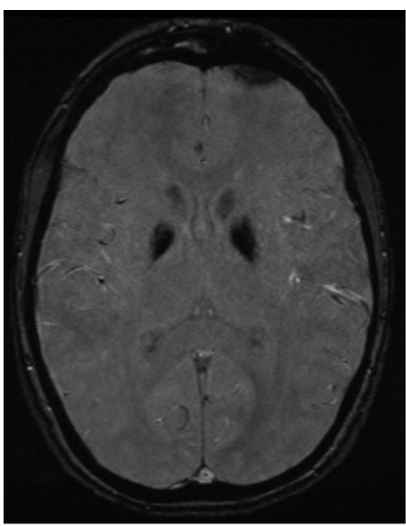

b.

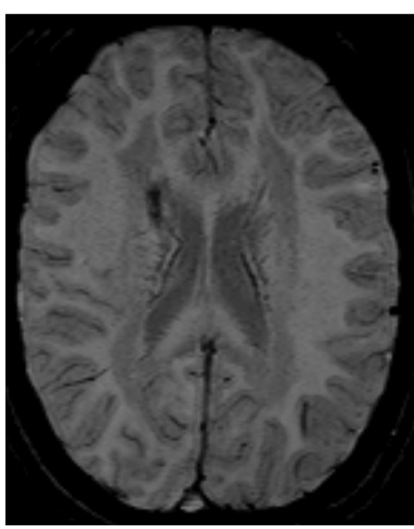

e.

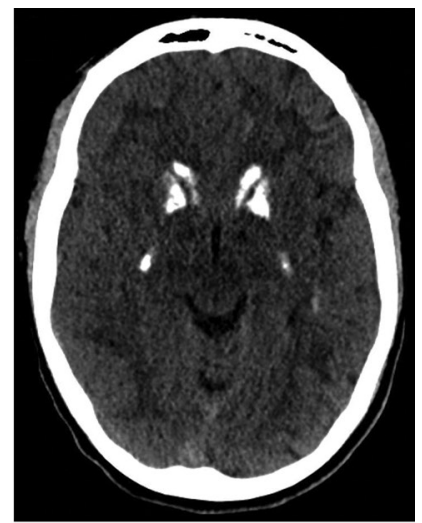

C.

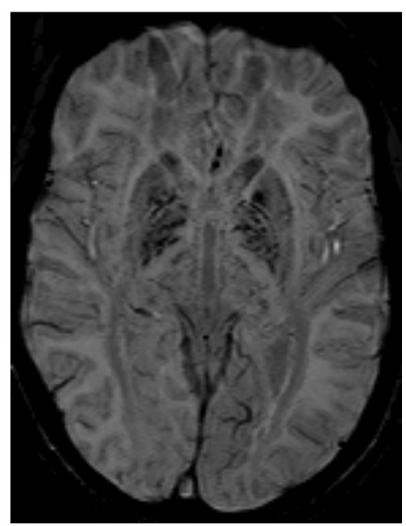

f.

Figure 16. Calcification in MELAS and Kearns-Sayre syndrome. (a, b) Axial CT (a) and susceptibilityweighted MR (b) images show parenchymal calcification involving the bilateral caudate nuclei and globi pallidi in a 13-year-old girl with a history of headaches and strokelike symptoms related to MELAS. (c-f) Axial CT (c, d) and susceptibility-weighted MR (e, f) images show parenchymal calcification involving the bilateral caudate nuclei and putamina in an 11-year-old boy with Kearns-Sayre syndrome.

\section{Spinal Cord Lesions}

Spinal cord involvement can be detected in several PMDs, the following disorders in particular: LBSL, Leigh syndrome, myoclonic epilepsy with ragged-red fibers, Kearns-Sayre syndrome, infantile-onset spinocerebellar ataxia, and pontocerebellar hypoplasia. Less commonly, spinal cord involvement is seen in association with MELAS, POLG-RD, and LHON. Patients may be found to have monoparesis, paraparesis, quadriparesis, sensory disturbances, hypotonia, spasticity, urinary or defecation dysfunction, spinal column deformities, or transverse myelitis-like syndrome (103).

\section{Increased Lactate Peak}

With PMDs, lactate abnormally and chronically accumulates in cells and tissues and can be detected at MR spectroscopy on the basis of a lactate peak at a chemical shift of $1.33 \mathrm{ppm}$, which may be superimposed by a lipid peak on short-echo-time images. This potential confusion can be resolved by applying an intermediate or long echo time (144 or $288 \mathrm{msec}$, respectively), which will mainly remove short-T2 components such as fat. In addition, applying an intermediate or long echo time, depending on the echo time used, may invert only the lactate peak, allowing more reliable detection (Fig 11e, 11f).

A lactate peak is not detected in all patients with PMDs, nor is it pathognomonic of PMDs, as it can be seen in several other metabolic disorders. A lactate peak can also be seen in necrotic tissues in the setting of ischemia, and in necrotic tumors or infectious processes such as abscesses. Significantly increased lactate peaks correlate with other markers of PMDs such as increased cerebrospinal fluid and serum lactate levels, and it may vary according to the type of PMD, the location of voxel sampling, and the temporal variation of disease involvement. MR spectroscopy is useful as a diagnostic tool and potentially as a follow-up examination for patients with PMDs since it may reveal metabolic abnormalities even when the brain parenchyma appears normal at conventional MRI (104).

\section{Succinate Peak}

MR spectroscopy may have a role in the diagnosis of succinate dehydrogenase deficiency or complex II disorder. Since a succinate peak is not 


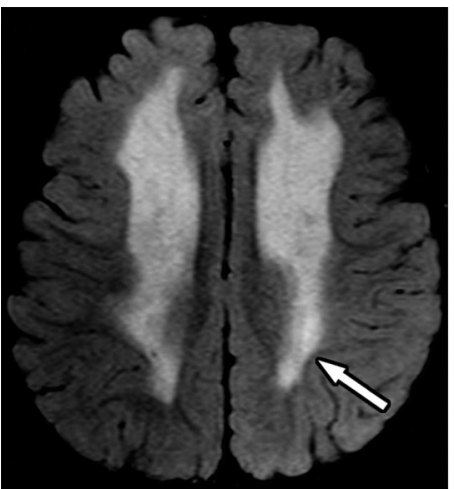

a.

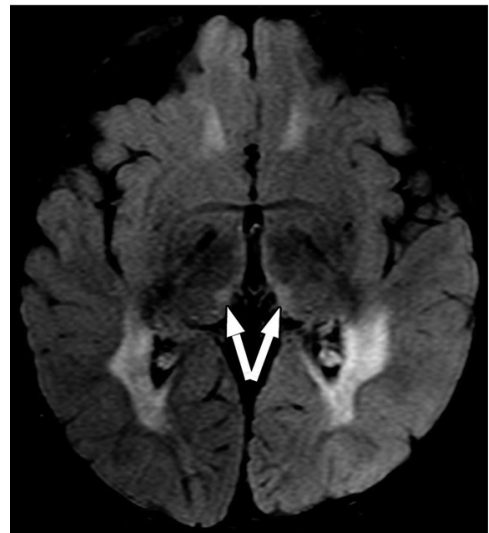

d.

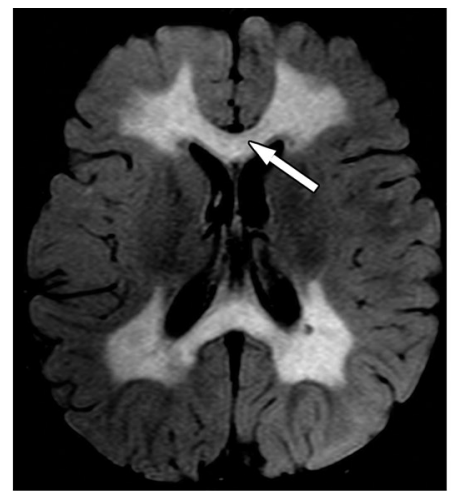

b.

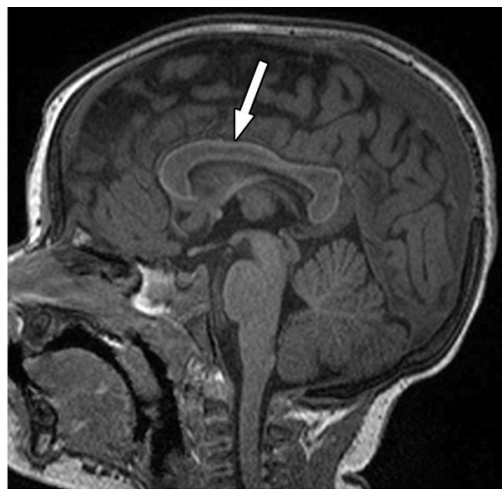

c.

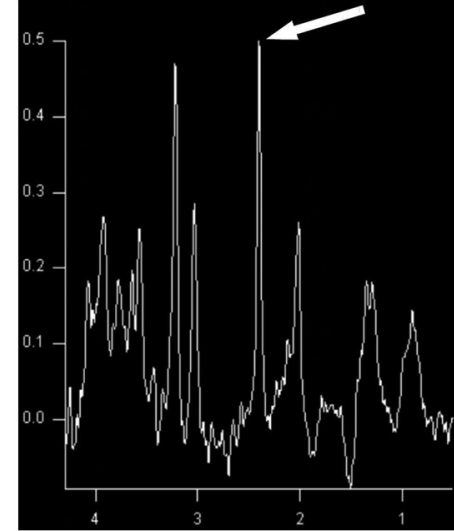

f.

Figure 17. Succinate dehydrogenase deficiency due to a homozygous SDHB gene variant in a 17-month-old boy with loss of developmental milestones. Axial FLAIR (a, b, d, e) and sagittal T1-weighted (c) MR images and MR spectroscopy image (f) show bilateral symmetric T2 white matter hyperintensity with sparing of the arcuate fibers (arrow in a) and associated diffuse corpus callosum swelling (arrow in b) with spared margins (arrow in c), bilateral medial thalamic changes (arrows in d), bilateral involvement of the middle cerebellar peduncles (arrows in e) and transverse pontine fibers, and a succinate peak at 2.40 ppm (arrow in $\mathbf{f}$ ).

seen in normal spectra, patients with succinate dehydrogenase deficiency or complex II disorder may have an apparent abnormal singlet peak at $2.40 \mathrm{ppm}$ in the cerebral and cerebellar white matter (Fig 17) (105).

\section{Hemorrhage}

Parenchymal hemorrhage is rarely described in association with PMDs. However, when hemorrhage does manifest with these disorders, it appears most frequently in adults. The literature on parenchymal hemorrhage in children is sparse. Recurrent brain hematomas were described in a pediatric patient with MELAS due to an ND5 gene variant (106). Intracerebral hemorrhage has been reported in a case of MERRF-MELAS overlap syndrome secondary to a variant in the $T 8356 C$ gene (107). Finally, a hemorrhagic putaminal lesion has been described in a patient with MERRF who was carrying the $A 8344 G$ mutation (108).

\section{Age at PMD Manifestation}

The patient's age at onset of the PMD should be considered relevant information when trying to narrow the differential diagnosis of PMDs. For example, PDH complex disorder frequently affects neonates. The Leigh syndrome phenotype typically manifests during the first year but may occur any time throughout childhood; even adult late onset has been described (109). MELAS and LBSL are more common during early and middle childhood. POLG-RD disorders can affect any age group, although the more severe phenotypes such as Alpers-Huttenlocher syndrome often occur during the first 5 years of life. In a male teenager with sudden vision loss and optic nerve thinning, there is high suspicion for LHON. Vision loss is almost always unilateral at presentation, with involvement of the other eye within months. Kearns-Sayre syndrome and CoQ10 deficiency can affect children of all age groups but primarily those in middle and late childhood.

\section{Conclusion}

PMDs display a profusion of imaging findings that significantly overlap with those of other non-PMD conditions. Therefore, patient age at 
onset, clinical symptoms, biochemical features, and imaging findings must be integrated into the diagnostic process to reach a unitary diagnosis or much narrower differential diagnosis.

Acknowledgments.-The authors thank Lydia Sheldon for proofreading and Brittany C. Bennett for creating the drawn illustrations.

Disclosures of Conflicts of Interest.-J.S.M.S. Activities related to the present article: disclosed no relevant relationships. Activities not related to the present article: paid postdoctoral research fellow, Department of Radiology, The Children's Hospital of Philadelphia. Other activities: disclosed no relevant relationships. A.G. Activities related to the present article: disclosed no relevant relationships. Activities not related to the present article: financial support from United Mitochondrial Disease Foundation and MitoAction for travel to meetings and presentations. Other activities: disclosed no relevant relationships.

\section{References}

1. BockFJ, Tait SWG. Mitochondria as multifaceted regulators of cell death. Nat Rev Mol Cell Biol 2020;21(2):85-100.

2. Chaban Y, Boekema EJ, Dudkina NV. Structures of mitochondrial oxidative phosphorylation supercomplexes and mechanisms for their stabilisation. Biochim Biophys Acta 2014;1837(4):418-426.

3. Gammage PA, Frezza C. Mitochondrial DNA: the overlooked oncogenome? BMC Biol 2019;17(1):53.

4. Calvo SE, Clauser KR, Mootha VK. MitoCarta2.0: an updated inventory of mammalian mitochondrial proteins. Nucleic Acids Res 2016;44(D1):D1251-D1257.

5. Calvo SE, Mootha VK. The mitochondrial proteome and human disease. Annu Rev Genomics Hum Genet 2010;11(1):25-44.

6. Holt IJ, Harding AE, Morgan-Hughes JA. Deletions of muscle mitochondrial DNA in patients with mitochondrial myopathies. Nature 1988;331(6158):717-719.

7. Wallace DC, Singh G, Lott MT, et al. Mitochondrial DNA mutation associated with Leber's hereditary optic neuropathy. Science 1988;242(4884):1427-1430.

8. McCormick EM, Zolkipli-Cunningham Z, Falk MJ. Mitochondrial disease genetics update: recent insights into the molecular diagnosis and expanding phenotype of primary mitochondrial disease. Curr Opin Pediatr 2018;30(6):714-724

9. Rahman J, Rahman S. Mitochondrial medicine in the omics era. Lancet 2018;391(10139):2560-2574.

10. Zhang H, Burr SP, Chinnery PF. The mitochondrial DNA genetic bottleneck: inheritance and beyond. Essays Biochem 2018;62(3):225-234.

11. Zolkipli-Cunningham Z, Xiao R, Stoddart A, et al. Mitochondrial disease patient motivations and barriers to participate in clinical trials. PLoS One 2018;13(5):e0197513.

12. Tanji K, Kunimatsu T, Vu TH, Bonilla E. Neuropathological features of mitochondrial disorders. Semin Cell Dev Biol 2001;12(6):429-439.

13. Filosto M, Tomelleri G, Tonin P, et al. Neuropathology of mitochondrial diseases. Biosci Rep 2007;27(1-3):23-30.

14. Shapira Y, Cederbaum SD, Cancilla PA, Nielsen D, Lippe BM. Familial poliodystrophy, mitochondrial myopathy, and lactate acidemia. Neurology 1975;25(7):614-621.

15. Betts J, Jaros E, Perry RH, et al. Molecular neuropathology of MELAS: level of heteroplasmy in individual neurones and evidence of extensive vascular involvement. Neuropathol Appl Neurobiol 2006;32(4):359-373.

16. Simonati A, Filosto M, Savio C, et al. Features of cell death in brain and liver, the target tissues of progressive neuronal degeneration of childhood with liver disease (Alpers-Huttenlocher disease). Acta Neuropathol (Berl) 2003;106(1):57-65.

17. Bourgeois M, Goutieres F, Chretien D, Rustin P, Munnich A, Aicardi J. Deficiency in complex II of the respiratory chain, presenting as a leukodystrophy in two sisters with Leigh syndrome. Brain Dev 1992;14(6):404-408.
18. Lake NJ, Compton AG, Rahman S, Thorburn DR. Leigh syndrome: one disorder, more than 75 monogenic causes. Ann Neurol 2016;79(2):190-203.

19. Sofou K, De Coo IFM, Isohanni P, et al. A multicenter study on Leigh syndrome: disease course and predictors of survival. Orphanet J Rare Dis 2014;9(1):52.

20. Schubert Baldo M, Vilarinho L. Molecular basis of Leigh syndrome: a current look. Orphanet J Rare Dis 2020;15(1):31. [Published correction appears in Orphanet J Rare Dis 2020;15(1):77.]

21. Fassone E, Rahman S. Complex I deficiency: clinical features, biochemistry and molecular genetics. J Med Genet 2012;49(9):578-590.

22. Rahman S, Blok RB, Dahl HH, et al. Leigh syndrome: clinical features and biochemical and DNA abnormalities. Ann Neurol 1996;39(3):343-351.

23. Wedatilake Y, Brown RM, McFarland R, et al. SURF1 deficiency: a multi-centre natural history study. Orphanet J Rare Dis 2013;8(1):96.

24. Alves CAPF, Teixeira SR, Martin-Saavedra JS, et al. Pediatric leigh syndrome: neuroimaging features and genetic correlations. Ann Neurol 2020;88(2):218-232. doi: 10.1002/ ana.25789. Published online May 22, 2020.

25. Assouline Z, Jambou M, Rio M, et al. A constant and similar assembly defect of mitochondrial respiratory chain complex I allows rapid identification of NDUFS4 mutations in patients with Leigh syndrome. Biochim Biophys Acta 2012;1822(6):1062-1069.

26. Rossi A, Biancheri R, Bruno C, et al. Leigh syndrome with COX deficiency and SURF1 gene mutations: MR imaging findings. AJNR Am J Neuroradiol 2003;24(6):1188-1191.

27. Copeland WC. The mitochondrial DNA polymerase in health and disease. Subcell Biochem 2010;50:211-222.

28. Cohen BH, Chinnery PF, Copeland WC. POLG -Related Disorders. In: Adam MP, Ardinger HH, Pagon RA, et al, eds. GeneReviews Seattle (WA). Seattle, Wash: University of Washington, 1993.

29. Darin N, Oldfors A, Moslemi AR, Holme E, Tulinius M. The incidence of mitochondrial encephalomyopathies in childhood: clinical features and morphological, biochemical, and DNA abnormalities. Ann Neurol 2001;49(3):377-383.

30. StumpfJD, Saneto RP, Copeland WC. Clinical and molecular features of POLG-related mitochondrial disease. Cold Spring Harb Perspect Biol 2013;5(4):a011395.

31. Hikmat O, Eichele T, Tzoulis C, Bindoff LA. Understanding the epilepsy in POLG related disease. Int J Mol Sci 2017;18(9):E1845.

32. Anagnostou ME, Ng YS, Taylor RW, McFarland R. Epilepsy due to mutations in the mitochondrial polymerase gamma (POLG) gene: a clinical and molecular genetic review. Epilepsia 2016;57(10):1531-1545.

33. Saneto RP, Lee IC, Koenig MK, et al. POLG DNA testing as an emerging standard of care before instituting valproic acid therapy for pediatric seizure disorders. Seizure 2010;19(3):140-146.

34. Gonçalves FG, Hill B, Guo Y, et al. The Perirolandic Sign: A Unique Imaging Finding Observed in Association with Polymerase $\gamma$-Related Disorders. AJNR Am J Neuroradiol 2020;41(5):917-922.

35. Yatsuga S, Povalko N, Nishioka J, et al. MELAS: a nationwide prospective cohort study of 96 patients in Japan Biochim Biophys Acta 2012;1820(5):619-624.

36. Chinnery PF, Turnbull DM. Epidemiology and treatment of mitochondrial disorders. Am J Med Genet 2001;106(1):94-101

37. Marin SE, Haas RH. Mitochondrial Myopathy, Encephalopathy, Lactic Acidosis, and Stroke-Like Episodes (MELAS). In: Saneto RP, Parikh S, Cohen BH, eds. Mitochondrial Case Studies: Underlying Mechanisms and Diagnosis. Amsterdam, the Netherlands: Elsevier, 2016; 13-29.

38. El-Hattab AW, Emrick LT, Hsu JW, et al. Impaired nitric oxide production in children with MELAS syndrome and the effect of arginine and citrulline supplementation. Mol Genet Metab 2016;117(4):407-412.

39. El-Hattab AW, Adesina AM, Jones J, Scaglia F. MELAS syndrome: clinical manifestations, pathogenesis, and treatment options. Mol Genet Metab 2015;116(1-2):4-12. 
40. Frederiksen AL, Andersen PH, Kyvik KO, Jeppesen TD, Vissing J, Schwartz M. Tissue specific distribution of the 3243A$>$ G mtDNA mutation. J Med Genet 2006;43(8):671-677.

41. Goto Y, Horai S, Matsuoka T, et al. Mitochondrial myopathy, encephalopathy, lactic acidosis, and stroke-like episodes (MELAS): a correlative study of the clinical features and mitochondrial DNA mutation. Neurology 1992;42(3 Pt 1):545-550.

42. Goodfellow JA, Dani K, Stewart W, et al. Mitochondrial myopathy, encephalopathy, lactic acidosis and stroke-like episodes: an important cause of stroke in young people. Postgrad Med J 2012;88(1040):326-334.

43. Kaufmann P, Engelstad K, Wei Y, et al. Natural history of MELAS associated with mitochondrial DNA m.3243A >G genotype. Neurology 2011;77(22):1965-1971.

44. Kolb SJ, Costello F, Lee AG, et al. Distinguishing ischemic stroke from the stroke-like lesions of MELAS using apparent diffusion coefficient mapping. J Neurol Sci 2003;216(1):11-15.

45. Allard JC, Tilak S, Carter AP. CT and MR of MELAS syndrome. AJNR Am J Neuroradiol 1988;9(6):1234-1238.

46. Kim IO, Kim JH, Kim WS, Hwang YS, Yeon KM, Han MC. Mitochondrial myopathy-encephalopathy-lactic acidosis-and strokelike episodes (MELAS) syndrome: CT and MR findings in seven children. AJR Am J Roentgenol 1996;166(3):641-645.

47. Ito $\mathrm{H}$, Mori $\mathrm{K}$, Harada $\mathrm{M}$, et al. Serial brain imaging analysis of stroke-like episodes in MELAS. Brain Dev 2008;30(7):483-488.

48. Liu Z, Zheng D, Wang X, et al. Apparent diffusion coefficients of metabolites in patients with MELAS using diffusion-weighted MR spectroscopy. AJNR Am J Neuroradiol 2011;32(5):898-902.

49. Hirano M, Pavlakis SG. Mitochondrial myopathy, encephalopathy, lactic acidosis, and strokelike episodes (MELAS): current concepts. J Child Neurol 1994;9(1):4-13.

50. Adam G, Ferrier M, Patsoura $S$, et al. Magnetic resonance imaging of arterial stroke mimics: a pictorial review. Insights Imaging 2018;9(5):815-831.

51. Zeviani M, Moraes CT, DiMauro S, et al. Deletions of mitochondrial DNA in Kearns-Sayre syndrome. Neurology 1988;38(9):1339-1346.

52. Obara-Moszynska M, Maceluch J, Bobkowski W, et al. A novel mitochondrial DNA deletion in a patient with KearnsSayre syndrome: a late-onset of the fatal cardiac conduction deficit and cardiomyopathy accompanying long-term rGH treatment. BMC Pediatr 2013;13(1):27.

53. Berardo A, DiMauro S, Hirano M. A diagnostic algorithm for metabolic myopathies. Curr Neurol Neurosci Rep 2010;10(2):118-126.

54. Schaefer AM, McFarland R, Blakely EL, et al. Prevalence of mitochondrial DNA disease in adults. Ann Neurol 2008;63(1):35-39.

55. Vázquez-Acevedo $M$, Vázquez-Memije ME, Mutchinick OM, Morales J, García-Ramos G, González-Halphen D. A case of Kearns-Sayre syndrome with the 4,977-bp common deletion associated with a novel 7,704-bp deletion. Neurol Sci 2002;23(5):247-250.

56. Yu M, Zhang Z, Wang QQ, et al. Clinical and Brain Magnetic Resonance Imaging Features in a Cohort of Chinese Patients with Kearns-Sayre Syndrome. Chin Med J (Engl) 2016;129(12):1419-1424.

57. Hourani RG, Barada WM, Al-Kutoubi AM, Hourani MH. Atypical MRI findings in Kearns-Sayre syndrome: T2 radial stripes. Neuropediatrics 2006;37(2):110-113.

58. Wray SH, Provenzale JM, Johns DR, Thulborn KR. MR of the brain in mitochondrial myopathy. AJNRAm J Neuroradiol 1995;16(5):1167-1173.

59. LivingstonJH, Stivaros S, Warren D, Crow YJ. Intracranial calcification in childhood: a review of aetiologies and recognizable phenotypes. Dev Med Child Neurol 2014;56(7):612-626.

60. Schiffmann R, van der Knaap MS. Invited article: an MRIbased approach to the diagnosis of white matter disorders. Neurology 2009;72(8):750-759.

61. Jurkute N, Yu-Wai-Man P. Leber hereditary optic neuropathy: bridging the translational gap. Curr Opin Ophthalmol 2017;28(5):403-409.
62. Mackey DA, Oostra RJ, Rosenberg T, et al. Primary pathogenic $\mathrm{mtDNA}$ mutations in multigeneration pedigrees with Leber hereditary optic neuropathy. Am J Hum Genet 1996;59(2):481-485.

63. Liu H, La Morgia C, Di Vito L, et al. Differences in onset between eyes in patients with Leber's hereditary optic neuropathy (LHON). Acta Ophthalmol 2017;95(S259).

64. Meyerson C, Van Stavern G, McClelland C. Leberhereditary optic neuropathy: current perspectives. Clin Ophthalmol 2015;9:1165-1176.

65. La Morgia C, Carbonelli M, Barboni P, Sadun AA, Carelli $\mathrm{V}$. Medical management of hereditary optic neuropathies. Front Neurol 2014;5:141.

66. Yu-Wai-Man P, Griffiths PG, Hudson G, Chinnery PF. Inherited mitochondrial optic neuropathies. J Med Genet 2009;46(3):145-158.

67. Mashima Y, Oshitari K, Imamura Y, Momoshima S, Shiga $\mathrm{H}$, Oguchi Y. Orbital high resolution magnetic resonance imaging with fast spin echo in the acute stage of Leber's hereditary optic neuropathy. J Neurol Neurosurg Psychiatry 1998;64(1):124-127.

68. Harrigan RL, Smith AK, Lyttle B, et al. Quantitative characterization of optic nerve atrophy in patients with multiple sclerosis. Mult Scler J Exp Transl Clin 2017;3(3):2055217317730097.

69. Yoshida T, Kido J, Mitsubuchi H, Matsumoto S, Endo F, Nakamura K. Clinical manifestations in two patients with pyruvate dehydrogenase deficiency and long-term survival. Hum Genome Var 2017;4(1):17020.

70. Barnerias C, Saudubray JM, Touati G, et al. Pyruvate dehydrogenase complex deficiency: four neurological phenotypes with differing pathogenesis. Dev Med Child Neurol 2010;52(2):e1-e9.

71. Lissens W, De Meirleir L, Seneca S, et al. Mutations in the X-linked pyruvate dehydrogenase (E1) alpha subunit gene (PDHA1) in patients with a pyruvate dehydrogenase complex deficiency. Hum Mutat 2000;15(3):209-219.

72. Patel KP, O'Brien TW, Subramony SH, Shuster J, Stacpoole PW. The spectrum of pyruvate dehydrogenase complex deficiency: clinical, biochemical and genetic features in 371 patients. Mol Genet Metab 2012;106(3):385-394.

73. Alves CAPF, Teixeira SR, Goncalves FG, Zuccoli G. Neuroimaging findings in primary mitochondrial cytopathies. In: Mancuso M, Klopstock T, eds. Diagnosis and management of mitochondrial disorders. Cham, Switzerland: Springer, 2019; 289-316.

74. Dallner G, Sindelar PJ. Regulation of ubiquinone metabolism. Free Radic Biol Med 2000;29(3-4):285-294.

75. Trevisson E, DiMauro S, Navas P, Salviati L. Coenzyme Q deficiency in muscle. Curr Opin Neurol 2011;24(5):449-456.

76. Goldstein AC, Bhatia P, Vento JM. Mitochondrial disease in childhood: nuclear encoded. Neurotherapeutics 2013;10(2):212-226.

77. Musumeci O, Naini A, Slonim AE, et al. Familial cerebellar ataxia with muscle coenzyme Q10 deficiency. Neurology 2001;56(7):849-855.

78. Poretti A, Wolf NI, Boltshauser E. Differential Diagnosis of Cerebellar Atrophy in Childhood: An Update. Neuropediatrics 2015;46(6):359-370.

79. van Berge L, Hamilton EM, Linnankivi T, et al. Leukoencephalopathy with brainstem and spinal cord involvement and lactate elevation: clinical and genetic characterization and target for therapy. Brain 2014;137(Pt 4):1019-1029.

80. Sissler M, Pütz J, Fasiolo F, Florentz C. Mitochondrial aminoacyl-tRNA synthetases. In: Madame Curie Bioscience Database [Internet]. Austin, Tex: Landes Bioscience; 2000-2013.

81. Yelam A, Nagarajan E, Chuquilin M, Govindarajan R. Leucoencephalopathy with brain stem and spinal cord involvement and lactate elevation: a novel mutation in the DARS2 gene. BMJ Case Rep 2019;12(1):bcr-2018-227755.

82. Steenweg ME, van Berge L, van Berkel CGM, et al. Early-onset LBSL: how severe does it get? Neuropediatrics 2012;43(6):332-338.

83. Steenweg ME, Pouwels PJW, Wolf NI, van Wieringen WN, Barkhof F, van der Knaap MS. Leucoencephalopathy with brainstem and spinal cord involvement and high lactate: 
quantitative magnetic resonance imaging. Brain 2011;134(Pt 11):3333-3341.

84. Yoon HJ, Kim JH, Jeon TY, Yoo SY, Eo H. Devastating metabolic brain disorders of newborns and young infants. RadioGraphics 2014;34(5):1257-1272.

85. Egloff C, Eldin de Pecoulas A, Mechler C, et al. Prenatal sonographic description of fetuses affected by pyruvate dehydrogenase or pyruvate carboxylase deficiency. Prenat Diagn 2018;38(8):607-616.

86. van Straaten HLM, van Tintelen JP, Trijbels JMF, et al Neonatal lactic acidosis, complex I/IV deficiency, and fetal cerebral disruption. Neuropediatrics 2005;36(3):193-199.

87. Saneto RP, Friedman SD, Shaw DWW. Neuroimaging of mitochondrial disease. Mitochondrion 2008;8(5-6):396-413.

88. Uusimaa J, Gowda V, McShane A, et al. Prospective study of POLG mutations presenting in children with intractable epilepsy: prevalence and clinical features. Epilepsia 2013;54(6):1002-1011

89. Farina L, Chiapparini L, Uziel G, Bugiani M, Zeviani M, Savoiardo M. MR findings in Leigh syndrome with COX deficiency and SURF-1 mutations. AJNR Am J Neuroradiol 2002;23(7):1095-1100.

90. Lee S, Na JH, Lee YM. Epilepsy in Leigh syndrome with mitochondrial DNA mutations. Front Neurol 2019;10:496.

91. Nakayama T, Wu J, Galvin-Parton P, et al. Deficient activity of alanyl-tRNA synthetase underlies an autosomal recessive syndrome of progressive microcephaly, hypomyelination, and epileptic encephalopathy. Hum Mutat 2017;38(10):1348-1354.

92. Fine AS, Nemeth CL, Kaufman ML, Fatemi A. Mitochondrial aminoacyl-tRNA synthetase disorders: an emerging group of developmental disorders of myelination. JNeurodev Disord 2019;11(1):29.

93. Xu W, Wen J, Sun C, Cao J, Li Y, Geng D. Conventional and Diffusional Magnetic Resonance Imaging Features of Mitochondrial Encephalomyopathy, Lactic Acidosis, and Stroke-Like Episodes in Chinese Patients: A Study of 40 Cases. J Comput Assist Tomogr 2018;42(4):510-516.

94. Ishiyama A, Muramatsu K, Uchino S, et al. NDUFAF3 variants that disrupt mitochondrial complex I assembly may associate with cavitating leukoencephalopathy. Clin Genet 2018;93(5):1103-1106.

95. Sue CM, Crimmins DS, Soo YS, et al. Neuroradiological features of six kindreds with MELAS tRNA(Leu) A2343G point mutation: implications for pathogenesis. J Neurol Neurosurg Psychiatry 1998;65(2):233-240.

96. Ardissone A, Tonduti D, Legati A, et al. KARS-related diseases: progressive leukoencephalopathy with brainstem and spinal cord calcifications as new phenotype and a review of literature. Orphanet J Rare Dis 2018;13(1):45.

97. Pirot N, Crahes M, Adle-Biassette H, et al. Phenotypic and neuropathological characterization of fetal pyruvate dehydrogenase deficiency. J Neuropathol Exp Neurol 2016;75(3):227-238

98. Vanlander AV, Van Coster R. Correction to: clinical and genetic aspects of defects in the mitochondrial iron-sulfur cluster synthesis pathway. J Biol Inorg Chem 2018;23(4):507.

99. Finsterer J, Zarrouk-Mahjoub S. Cerebral imaging in paediatric mitochondrial disorders. Neuroradiol J 2018;31(6):596-608.

100. Rossignol DA, Frye RE. Mitochondrial dysfunction in autism spectrum disorders: a systematic review and meta-analysis. Mol Psychiatry 2012;17(3):290-314.

101. Finsterer J, Zarrouk-Mahjoub S. Cerebellar atrophy is common among mitochondrial disorders. Metab Brain Dis 2018;33(4):987-988.

102. Mori O, Yamazaki M, Ohaki Y, et al. Mitochondrial encephalomyopathy with lactic acidosis and stroke like episodes (MELAS) with prominent degeneration of the intestinal wall and cactus-like cerebellar pathology. Acta Neuropathol (Berl) 2000;100(6):712-717.

103. Finsterer J, Zarrouk-Mahjoub S. Involvement of the spinal cord in mitochondrial disorders. J Neurosci Rural Pract 2018;9(2):245-251.

104. Ohlenbusch A, Edvardson S, Skorpen J, et al. Leukoencephalopathy with accumulated succinate is indicative of SDHAF1 related complex II deficiency. Orphanet J Rare Dis 2012;7(1):69.

105. Helman G, Caldovic L, Whitehead MT, et al. Magnetic resonance imaging spectrum of succinate dehydrogenaserelated infantile leukoencephalopathy. Ann Neurol 2016;79(3):379-386.

106. Pénisson-Besnier I, Reynier P, Asfar P, et al. Recurrent brain hematomas in MELAS associated with an ND5 gene mitochondrial mutation. Neurology 2000;55(2):317-318.

107. Kato H, Uchigata M, Iijima M, Shimizu S, Nonaka I, Goto Y. Fatal cerebral hemorrhage in mitochondrial encephalomyopathy: clinical and pathological data of a case. J Neurol 2006;253(4):529-530.

108. Huang CC, Kuo HC, Chu CC, Liou CW, Ma YS, Wei $\mathrm{YH}$. Clinical phenotype, prognosis and mitochondrial DNA mutation load in mitochondrial encephalomyopathies. J Biomed Sci 2002;9(6 Pt 1):527-533.

109. McKelvie P, Infeld B, Marotta R, Chin J, Thorburn D, Collins S. Late-adult onset Leigh syndrome. J Clin Neurosci 2012;19(2):195-202. 